\title{
DIGITALCOMMONS
}

$@$ WAYNESTATE-

Wayne State University

$1-1-2010$

\section{Optimal Control and Feedback Design of State- Constrained Parabolic Systems in Uncertainty Conditions}

Boris S. Mordukhovich

Wayne State University, boris@math.wayne.edu

\section{Recommended Citation}

Mordukhovich, Boris S., "Optimal Control and Feedback Design of State-Constrained Parabolic Systems in Uncertainty Conditions" (2010). Mathematics Research Reports. Paper 72.

http://digitalcommons.wayne.edu/math_reports/72

This Technical Report is brought to you for free and open access by the Mathematics at DigitalCommons@WayneState. It has been accepted for inclusion in Mathematics Research Reports by an authorized administrator of DigitalCommons@WayneState. 
OPTIMAL CONTROL AND FEEDBACK DESIGN OF STATE-CONSTRAINED PARABOLIC SYSTEMS IN UNCERTAINTY CONDITIONS

\author{
BORIS S. MORDUKHOVICH
}

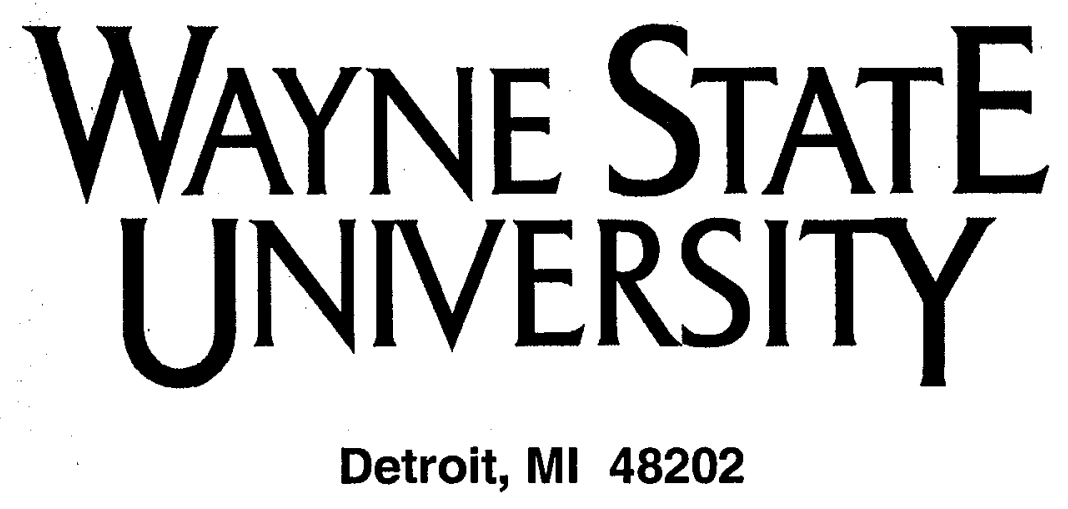

Department of Mathematics

Research Report

2010 Series

\#1

This research was partly supported by the USA National Science Foundation 


\title{
OPTIMAL CONTROL AND FEEDBACK DESIGN OF STATE-CONSTRAINED PARABOLIC SYSTEMS IN UNCERTAINTY CONDITIONS
}

\author{
BORIS S. MORDUKHOVICH ${ }^{1}$
}

\begin{abstract}
The paper concerns minimax control problems for linear multidimensional parabolic systems with distributed uncertain perturbations and control functions acting in the Dirichlet boundary conditions. The underlying parabolic control system is functioning under hard/pointwise constraints on control and state variables. The main goal is to design a feedback control regulator that ensures the required state performance and robust stability under any feasible perturbations and minimize an energy-type functional under the worst perturbations from the given area. We develop a constructive approach to the minimax control design of constrained parabolic systems that is based on certain characteristic features of the parabolic dynamics including the transient monotonicity with respect to both controls and perturbations and the turnpike asymptotic behavior on the infinite horizon. In this way, solving a number of associated open-loop control and optimization problems, we justify an easily implementable three-positional suboptimal structure of the feedback boundary regulator and compute its optimal parameters, ensuring thus the required state performance and robust stability of the closed-loop, highly nonlinear parabolic control system on the infinite horizon.
\end{abstract}

Key words. parabolic systems, Dirichlet boundary controls, state constraints, uncertainty perturbations, feedback control, suboptimality, minimax synthesis, robust stability

AMS subject classifications. 49K20, 49K35, 49N35, 93B50, 93D09

\section{Introduction and Problem Formulation}

This paper is devoted to developing an constructive procedure to design a suboptimal feedback control regulator acting in the Dirichlet boundary conditions of a multidimensional linear parabolic system with hard/pointwise constraints on the state and control variables under distributed uncertain perturbations. Problems of this type are among the most challenging and difficult in control theory while being among the most important for various applications. The original motivation for our development came from practical design problems of automatic control of the soil groundwater regime in irrigation engineering networks functioning under uncertain weather and environmental conditions; see [12] for technological descriptions and modeling.

The system dynamics in the problem under consideration is given by the multidimensional linear parabolic equation

$$
\left\{\begin{array}{l}
\frac{\partial y}{\partial t}+A y=w(t) \quad \text { a.e. in } Q:=[0, T] \times \Omega \\
y(0, x)=0, \quad x \in \Omega \\
y(t, x)=u(t), \quad(t, x) \in \Sigma:=[0, T] \times \partial \Omega
\end{array}\right.
$$

\footnotetext{
${ }^{1}$ Department of Mathematics, Wayne State University, Detroit, Michigan 48202 (boris@math.wayne.edu). This research was partly supported by the National Science Foundation under grant DMS-0603846.
} 
with controls $u(\cdot)$ acting in the Dirichlet boundary conditions and distributed perturbations $w(\cdot)$ on the right-hand side of the parabolic equation. In (1.1), $A$ is a self-adjoint and uniformly strongly elliptic operator on $L^{2}(\Omega)$ defined by

$$
A y:=-\sum_{i, j=1}^{n} \frac{\partial}{\partial x_{i}}\left(a_{i j}(x) \frac{\partial y}{\partial x_{j}}\right)-c y
$$

(see Section 2 for the precise assumptions), where $\Omega \subset \mathbb{R}^{n}$ is an open bounded domain with the closure $c] \Omega$ and the boundary $\partial \Omega$ that is supposed to be a sufficiently smooth $(n-1)$-dimensional manifold, and where $T>0$ is a fixed time bound.

The sets of admissible controls $U$ and admissible perturbations $W$ are given, respectively, by the relationships

$$
\begin{aligned}
& U:=\left\{u \in L^{\infty}[0, T] \mid-\alpha \leq u(t) \leq \alpha \text { a.e. } t \in[0, T]\right\} \\
& W:=\left\{w \in L^{\infty}[0, T] \mid-\beta \leq w(t) \leq \beta \text { a.e. } t \in[0, T]\right\}
\end{aligned}
$$

with some fixed bounds $\alpha, \beta>0$. Note that control and perturbation functions look similarly via the pointwise constraints in (1.3) and (1.4)-except they are situated in the different parts of the parabolic system (1.1)--while their roles in the feedback control problem formulated below are completely opposite.

It has been well recognized that the Dirichlet boundary conditions as in (1.1) offer the least regularity properties of the parabolic dynamics and occur to be the most challenging in control theory; see, e.g., $[3,9,14,19,21]$ with various results, discussions, and references therein. In particular, a lower regularity of feasible controls in (1.3) is not sufficient for the existence of classical solutions to the initial-boundary value problem in (1.1), while for any feasible pair $(u, w) \in U \times W$ there is a unique generalized solution $y \in L^{2}(Q)$ to the parabolic system (1.1); see, e.g., [10]. Having this in mind, fix a point $x_{0} \in \Omega$ from the space domain and suppose that we are able to collect information about the system motion/performance $y\left(t, x_{0}\right)$ at this point. Since the domain $\Omega$ is open and $u, w \in L^{\infty}[0, T]$, we can pointwisely evaluate $y\left(t, x_{0}\right)$ for any $x_{0} \in \Omega$; see, e.g., [1, Theorem 3.9].

A crucial requirement on the system performance (originally motivated by the groundwater control problem in [12]) is to keep the motion $y\left(t, x_{0}\right)$ within the given distance $\eta>0$ from the initial equilibrium state $y(x, 0) \equiv 0$ for the whole dynamic process. This means imposing the pointwise state constraints on the motion under observation

$$
-\eta \leq y\left(t, x_{0}\right) \leq \eta \text { a.e. } t \in[0, T] .
$$

As mentioned, perturbations $w(\cdot)$ in (1.1) are uncertain, i.e., they are not known a priori; the only information available on perturbations is the bound $\beta$ of their admissible variations. The main goal of boundary controls $u(\cdot)$ in (1.1) is to keep the motion $y\left(t, x_{0}\right)$ within the state constraints (1.5) for all admissible perturbations $w(\cdot)$ from (1.4). Clearly, it cannot be done in any (prescribed) open loop $u=u(t)$, and so control actions in the boundary conditions of (1.1) should be formed depending on the current position $y\left(t, x_{0}\right)$ under observation. This means that we have to design a feedback control regulator in the boundary conditions as a function of the state position $\xi \in \mathbb{R}^{n}$, where $\xi$ is generated by the dynamic system (1.1) via the moving point of observation $y\left(t, x_{0}\right)$ for each $t \in[0, T]$. 
To formalize this procedure, we consider a function $f: \mathbb{R} \rightarrow \mathbb{R}$ satisfying the composite summability condition

$$
|f(\gamma(t))| \in L^{1}[0, T] \text { whenever } \gamma(t) \in L^{2}[0, T]
$$

and construct boundary controls in (1.1) via the feedback law

$$
u(t):=f\left(y\left(t, x_{0}\right)\right), \quad t \in[0, T] .
$$

Thus boundary controls $u(t)$ in (1.1) are fully determined via (1.7) by the choice of a feedback function/regulator $f=f(\xi)$. We say that such a function $f$ defines a feasible regulator if it satisfies the summability condition (1.6), generates controls $u(t)$ by (1.7) belonging to the admissible set $U$ from (1.3), and keeps the corresponding motions $y\left(t, x_{0}\right)$ of the parabolic system (1.1) within the prescribed constraint area (1.5) for every admissible perturbation $w \in W$ from (1.4). The set of all feasible regulators is labeled as $\mathcal{F}$. We refer the reader to [17] for efficient solvability conditions ensuring that $\mathcal{F} \neq \emptyset$.

To estimate the quality of feasible regulators $f=f(\xi)$, we consider the cost functional

$$
J(f):=\max _{w \in W}\left\{\int_{0}^{T}\left|f\left(y\left(t, x_{0}\right)\right)\right| d t\right\}
$$

which is an energy-type functional with respect to controls (1.7) in the boundary conditions of (1.1) subject to the symmetric constraints (1.3). The maximum operation in (1.8) reflects the required control energy needed to neutralize the adverse effect of the worst perturbations from (1.4) and to keep the state performance within the prescribed area (1.5).

The minimax feedback control problem $(P)$ studied in this paper is as follows:

$$
\text { minimize } J(f) \text { over } f \in \mathcal{F} \text {, }
$$

i.e., to find an optimal feedback control $\bar{f}=\bar{f}(\xi)$ that minimizes the energy-type cost functional (1.8) over the set $\mathcal{F}$ of all feasible regulators, provided of course that $\mathcal{F} \neq \emptyset$.

It has been well recognized in control theory and applications that feedback control problems are the most challenging and important for any type of dynamical systems, while PDE systems provide additional difficulties and much less investigated in comparison, e.g., with the ODE dynamics; see more discussions and references in [14]. Furthermore, significant complications come from pointwise/hard constraints on control and (much more) state functions; the latter are of high nontriviality even for open-look control problems, especially in the case of Dirichlet boundary control (see, in particular, the aforementioned publications $[3,19,14,21])$. We are not familiar with any device applicable to the problem $(P)$ under consideration among a variety of approaches and results available in the theories of differential games, $H_{\infty}$-control, Riccati's feedback synthesis, etc.; see, e.g., $[2,6,7,9]$ and the references therein.

In this paper we develop and significantly extend the approach to solving the feedback control problem $(P)$, which was initiated in [13] for the case of the one-dimensional heat equation in $(1.1)$; see also $[14,16]$ for partial results reported for Dirichlet boundary controls of multidimensional parabolic systems and [15] for the cases of controls in the Neumann and mixed (Robin) boundary conditions.

Our approach is essentially based on certain underlying features of the parabolic dynamics, particularly on the monotonicity property of transients, which is eventually related 
to the fundamental Maximum Principle for parabolic equations; see Section 2. Due to this property and the specific structures of the cost functional (1.8) and boundary controls in (1.1), we are able to select the worst perturbations in the area (1.4) for the class of nonincreasing and odd feedbacks (1.7) and then to study the corresponding open-loop optimal control problem with pointwise state constraints as a reaction of the parabolic system to the worst perturbations. Using the spectral Fourier type representation of solutions to the parabolic system (1.1) and assuming the positivity of the first eigenvalue of the elliptic operator $A$ in (1.2)-which is often the case- we observe the dominance of the first term in the exponential series representation of solutions to (1.1) as $t \rightarrow \infty$. This allows us to justify an efficient approximation of the open-loop optimal control problem for the parabolic system under consideration by that for the corresponding $O D E$ system with state constraints on a sufficiently large time interval. Moreover, the approximating ODE optimal control problem is solved exactly-under some requirements on the initial data of $(P)$ - by constructing yet another approximation of state constraints, employing the Pontryagin maximum principle that provides necessary and sufficient optimality conditions for the unconstrained approximating problems with both bang-bang and singular modes of optimal controls, and then by passing to the limit while meeting the state constraints. It happens in this way (due to specific features of the ODE problems under consideration approximating the parabolic dynamics) that the state constraints surprisingly occur to be a regularization factor, which simplifies the structure of optimal controls, especially when the time interval becomes bigger and bigger $(T \rightarrow \infty)$-this reveals the fundamental turnpike property of such dynamic systems expanding to the infinite horizon.

Thus using the ODE approximation described above, we justify an easily implementable suboptimal (or near-optimal) structures of optimal controls in both open-loop and closedloop modes and then optimize their parameters along the parabolic dynamics. This allows us arrive at a three-positional feedback regulator $f=f(\xi)$ in (1.7) acting via the Dirichlet boundary conditions of (1.1) that ensures the required state performance (1.5) under the fulfillments of all the constraints in $(P)$ for every feasible perturbation from (1.4) providing a near-optimal response of the closed-loop control system in the case of worst perturbations.

The feedback control design constructed in this way leads us to the highly nonlinear closed-loop system (1.1) and (1.7), where $f(\xi)$ is a discontinuous three-positional regulator. The system may loose robust stability (in the large) and maintain the state performance (1.5) in a unacceptable self-vibrating regime. Developing a variational approach to robust stability that reduces the stability issue to a certain open-loop optimal control problem on the infinite horizon, we establish efficient conditions for robust stability of the closed-loop system whenever $t \geq 0$ in terms of the initial data of problem $(P)$ and parameters of the three-positional feedback regulator.

The rest of the paper is organized as follows. In Section 2 we formulate the standing assumptions on the parabolic system (1.1) and then present efficient conditions ensuring the fulfillment of these assumptions and also certain important properties of the parabolic dynamics following from them.

Section 3 includes the underlying monotonicity property of solutions to (1.1) with respect to both controls and perturbations, which is a consequence of the fundamental Maximum Principle for the parabolic dynamics. Based on this result and on the specific features of the minimax problem $(P)$ from (1.9), we justify the selection of the worst perturbations $(P)$ as the extreme values in (1.4) for every odd and nonincreasing feedback law $f(\xi)$ in (1.7).

This allows us to consider next an open-loop parabolic control problem arising from $(P)$ 
under the worst/extreme perturbations and then to approximate it by appropriate $O D E$ systems subject to pointwise state and control constraints. Observing that the first-order approximation is sufficiently adequate to the parabolic dynamic on large time intervals (due to the aforementioned first-term dominance in the spectral representation of trajectories), we concentrate in Section 4 on the corresponding state-constrained ODE optimal control problem and solve it completely by using a penalty-type approximation of the state constraints and the Pontryagin maximum principle providing in this case necessary and sufficient conditions for the open-loop control optimality.

In Section 5 we take the optimal control structure computed for the ODE constrained problem in Section 4 and impose it as a suboptimal structure of open-loop boundary controls for the parabolic system (1.1) acting under the worst perturbations. Furthermore, we optimize the parameters of this structure along the constrained parabolic dynamics.

Section 6 is devoted to computing parameters of the minimax control design for the parabolic system (1.1) with feedback controls of type (1.7) defining by nonincreasing and odd functions $f(\xi)$. We justify the structure of a three-positional feedback regular and compute its parameters in such a way that it gives the open-loop (sub)optimal control realization of Section 4 for the case of worst perturbations while keeping the dynamics within the prescribed constraints (1.5) for any feasible perturbation $w \in W$.

In Section 7 we compute optimal parameters of the closed-loop nonlinear control system from Section 6, which ensure robust stability of the stabilizing equilibrium state of the system for all $t>0$. This is done by reducing the stability issue to an open-loop constrained optimal control problem on the infinite horizon. Finally, we establish verifiable reliability conditions for the feedback control design that simultaneously ensures controllability, stability, and minimax optimality of the closed-loop constrained parabolic system.

\section{Standing Assumptions and Preliminary Results}

Consider the parabolic system (1.1) with the operator $A$ defined in (1.2), where $c \in \mathbb{R}$ and where the functions $a_{i j}: \operatorname{cl} \Omega \rightarrow \mathbb{R}$ satisfy the properties:

$$
\begin{aligned}
& a_{i j} \in C^{\infty}(\operatorname{cl} \Omega), \quad a_{i j}(x)=a_{j i}(x) \text { for all } x \in \Omega, \quad i, j=1, \ldots, n, \\
& \sum_{i, j=1}^{n} a_{i j}(x) v_{i} v_{j} \geq \nu \sum_{i=1}^{n} v_{i}^{2} \text { with some } \nu>0
\end{aligned}
$$

whenever $x \in \Omega$ and $v=\left(v_{1}, \ldots, v_{n}\right) \in \mathbb{R}^{n}$. Then the differential operator (1.2) is selfadjoint and uniformly strongly elliptic on $L^{2}(\Omega)$.

Observe that the input data $(u, w) \in L^{\infty}[0, T] \times L^{\infty}[0, T]$ are irregular to ensure the existence of the classical smooth solutions $y$ to (1.1). Nevertheless, for all admissible pairs $(u, w) \in U \times W$ system (1.1) admits a unique generalized solution $y=y(t, x) \in L^{2}(Q)$; this is proved, e.g., in [10]. In what follows we present a convenient series representation of generalized solutions to the parabolic equation (1.1) generated by admissible pairs $(u, w)$, while first we discuss some properties of uniformly strongly elliptic operators crucial for establishing the main results of the paper.

Given the operator $A$ in (1.2), consider the homogeneous boundary value problem

$$
\left\{\begin{array}{l}
-A \phi+\lambda \phi=0 \\
\left.\phi\right|_{\partial \Omega}=0
\end{array}\right.
$$


and recall that the number component $\lambda$ in the nontrivial pair $(\lambda, \phi)$ satisfying (2.2) is an eigenvalue, while $\phi$ is the corresponding eigenfunction for the operator $A$ under the Dirichlet boundary condition. According to [4, Theorems 8.37, 8.38], the assumptions imposed on the operator $A$ in (2.1) ensure the following properties:

(a) The eigenvalues $\lambda_{i}, i=1,2, \ldots$, are real and form a nondecreasing sequence, which accumulates only at $\infty$;

(b) The corresponding orthonormal system $\left\{\phi_{i}(x)\right\} \subset C^{\infty}(\Omega)$ of eigenfunctions is complete in $L^{2}(\Omega)$.

(c) The first eigenvalue $\lambda_{1}$ is simple and has the positive eigenfunction $\phi_{1}(x)$.

In addition to these underlying properties, the next proposition offers one more important consequence of the strong ellipticity.

Proposition 2.1 (consequence of strong ellipticity ). Let $\lambda$ be an eigenvalue of the operator $A$ in (1.2) satisfying the assumptions in (2.1). Then for any constant $c \in \mathbb{R}$ in (1.2) the sum $c+\lambda$ is positive.

Proof. It follows from [11, Theorem 3.20] that $c+\lambda \geq 0$. Thus it remains to show that $c+\lambda \neq 0$. Assuming the contrary, i.e., that $c+\lambda=0$ and substituting the latter into (2.2), we get that the eigenfunction $\phi$ corresponding to $\lambda$ is a solution to the homogeneous elliptic boundary value problem

$$
\left\{\begin{array}{l}
\sum_{i=1}^{n} \frac{\partial}{\partial x_{i}}\left(a_{i j} \frac{\partial \phi}{\partial x_{j}}\right)=0 \\
\left.\phi\right|_{\partial \Omega}=0
\end{array}\right.
$$

Due to the well-known uniqueness of solutions to the latter problem, we have that $\phi \equiv 0$, which contradicts the above properties (a) and (c) and completes the proof.

In addition to (2.1), the basis hypothesis needed to develop our approach is as follows:

(H) The first/principal eigenvalue $\lambda_{1}$ of the operator $A$ in (1.2) is positive.

Along with properties (a) and (c), the latter implies that the eigenvalues of $A$ satisfy the series of inequalities

$$
0<\lambda_{1}<\lambda_{2} \leq \lambda_{3} \leq \ldots \leq \ldots
$$

Let us provide an efficient condition on parameters of the operator $A$ in connection with the size of the domain $\Omega$ that ensures the fulfillment of (2.3).

Proposition 2.2 (positivity of the first eigenvalue). Let

$$
d:=\sup _{x_{1}, x_{2} \in \Omega}\left\|x_{1}-x_{2}\right\|
$$

be the diameter of the domain $\Omega \subset \mathbb{R}^{n}$ in (1.1), and let the relationship

$$
c<\frac{2 n \nu}{d^{2}}
$$

hold for the parameters of the operator $A$ from (1.2), where $\nu>0$ is the constant of strong ellipticity in (2.1). Then $\lambda_{1}>0$ for the first eigenvalue of $A$. 
Proof. Let the pair $(\lambda, \phi)$ satisfy (2.2). Multiplying the equation in (2.2) by $\phi$ and then integrating by parts, we come up to the equality

$$
(c+\lambda) \int_{\Omega} \phi^{2}(x) d x=\int_{\Omega} \sum_{i, j=1}^{n} a_{i j}(x) \frac{\partial \phi}{\partial x_{i}}(x) \frac{\partial \phi}{\partial x_{j}}(x) d x .
$$

The latter implies, due to the strong ellipticity in (2.1), that

$$
(c+\lambda)\|\phi(x)\|^{2} \geq \nu \sum_{i=1}^{n}\left\|\frac{\partial \phi}{\partial x_{i}}(x)\right\|^{2} .
$$

Taking Proposition 2.1 into account and employing the Poincaré inequality (see, e.g., [11, Lemma 3.3]) in (2.5), we get

$$
\lambda \geq \frac{2 n \nu}{d^{2}}-c>0
$$

by (2.4), which ensures that $\lambda_{1}>0$ and thus completes the proof.

As mentioned above, for every feasible input pair $(u, w) \in L^{\infty}[0, T] \times L^{\infty}[0, T]$ the parabolic system (1.1) admits a unique generalized solution $y \in L^{2}(Q)$. The next proposition gives a convenient spectral representation of this solution via a Fourier-like series involving the eigenvalues and eigenfunctions $\left(\lambda_{i}, \phi_{i}\right)$ of the operator $A$. This representation, which is widely used in what follows, is essentially related to the given setting of system (1.1), where both Dirichlet boundary controls $u=u(t)$ and distributed perturbations $w=w(t)$ are spatially constant, i.e., independent of state variables.

Proposition 2.3 (spectral representation of transients). Let $(u, w) \in L^{2}[0, T] \times$ $L^{2}[0, T]$ in (1.1) under assumptions (2.1) on the strongly elliptic operator $A$, and let $\left(\lambda_{i}, \phi_{i}\right)$ be the corresponding eigenvalues and eigenfunctions of $A$ with the weights

$$
\mu_{i}:=\int_{\Omega} \phi_{i}(x) d x, \quad i=1,2, \ldots
$$

Then the unique solution $y \in L^{2}(Q)$ to (1.1) admits the spectral representation

$$
y(t, x)=\sum_{i=1}^{\infty} \mu_{i}\left(\int_{0}^{t} w(\theta) e^{\lambda_{i} \theta} d \theta+\left(c+\lambda_{i}\right) \int_{0}^{t} u(\theta) e^{\lambda_{i} \theta} d \theta\right) e^{-\lambda_{i} t} \phi_{i}(x),
$$

where the series in (2.6) strongly converges in the space $L^{2}(Q)$.

Proof. Let $\mathcal{D}[0, T]$ be the space of $C^{\infty}$-functions on $[0, T]$ with compact supports. Since $\mathcal{D}[0, T]$ is dense in $L^{2}[0, T]$, we pick sequences $\left\{u_{k}\right\}$ and $\left\{w_{k}\right\}$ from $\mathcal{D}[0, T]$ such that

$$
\left(u_{k}, w_{k}\right) \rightarrow(u, w) \text { strongly in } L^{2}[0, T] \times L^{2}[0, T] \text { as } k \rightarrow \infty .
$$

It is well known (see, e.g., [8]) that system (1.1) has a unique classical solution $y_{k}$ for each pair $\left(u_{k}, w_{k}\right) \in \mathcal{D}[0, T] \times \mathcal{D}[0, T]$. Furthermore, for each $(u, w) \in L^{2}[0, T] \times L^{2}[0, T]$ it admits a unique generalized solution $y \in L^{2}(Q)$ in the sense of $[10]$, where it is proved that the linear operator $(u, w) \rightarrow y$ from $L^{2}[0, T] \times L^{2}[0, T]$ into $L^{2}(Q)$ is continuous. Combining this with the strong convergence in (2.7), we get

$$
y_{k} \rightarrow y \text { strongly in } L^{2}(Q) \text { as } k \rightarrow \infty \text {. }
$$


Consider now the sequence $\left\{\left(u_{k}, w_{k}\right)\right\}$ from (2.7) and denote $h_{k}:=y_{k}-u_{k}$ for each $k=1,2, \ldots$. We can easily conclude that $h_{k}$ is the unique classical solution to the system

$$
\left\{\begin{array}{l}
\frac{\partial h}{\partial t}+A h=w_{k}(t)-u_{k}^{\prime}(t)+c u_{k}(t) \\
h(0, x)=0, \quad x \in \Omega \\
h(t, x)=0, \quad(t, x) \in \Sigma
\end{array}\right.
$$

with the homogeneous Dirichlet boundary conditions. It is well known in the classical parabolic theory (see, e.g., [8]) that $h_{k}$ admits the representation

$$
\begin{aligned}
h_{k}(t, x)= & \sum_{i=1}^{\infty} \mu_{i}\left(\int_{0}^{t} w_{k}(\theta) e^{\lambda_{i} \theta} d \theta+\left(c+\lambda_{i}\right) \int_{0}^{t} u_{k}(\theta) e^{\lambda_{i} \theta} d \theta\right) e^{-\lambda_{i} t} \phi_{i}(x) \\
& -u_{k}(t) \sum_{i=1}^{\infty} \mu_{i} \phi_{i}(x) \text { for each } k=1,2 \ldots
\end{aligned}
$$

Since one obviously has $h_{k}+u_{k}=y_{k}$ for each $k \in \mathbb{N}$ and since

$$
\sum_{i=1}^{\infty} \mu_{i} \phi_{i}(x)=1
$$

with the strong convergence in $L^{2}(\Omega)$ (by the orthonormality property of $\left\{\phi_{i}(x)\right\}$ in $L^{2}(\Omega)$ and the construction of $\mu_{i}$ in the theorem), we conclude that the triple $\left(u_{k}, w_{k}, y_{k}\right)$ satisfies (2.6) for all $k=1,2, \ldots$. Passing there to the limit as $k \rightarrow \infty$ with taking (2.7) and (2.8) into account, we arrive at the limiting representation (2.6) for the reference triple $(u, w, y) \in L^{2}[0, T] \times L^{2}[0, T] \times L^{2}(Q)$ and thus conclude the proof of the proposition.

\section{Dynamics Monotonicity and Worst Perturbations}

In this section we begin our study of the minimax feedback control problem $(P)$ formulated in (1.9) assuming that it has at least one feasible solution, i.e., $\mathcal{F} \neq \emptyset$. Observe that it is not always the case; see, in particular, counterexamples as well positive results in [17], where this issue is considered from the viewpoint of asymmetric games.

A major fact established in this section is proving a monotonicity property of transients with respect to both control and perturbations, which is largely due to the fundamental Maximum Principle for the parabolic dynamics. This fact, together with the convolution representation of transients obtained in [17], allow us to justify the choice of the worst perturbations, for feedback laws described by odd and nonincreasing functions $f(\xi)$ in (1.7), as those taking the extreme values in the feasible perturbation region (1.4). The latter in turn gives us a possibility to properly decompose the minimax problem and to develop a constructive approach to the minimax control design starting with the open-loop system reaction to the revealed worst perturbations.

We first formulate and prove the aforementioned monotonicity property of transients.

Theorem 3.1 (monotonicity property of the parabolic dynamics). Let $\left(u_{1}, w_{1}\right)$ and $\left(u_{2}, w_{2}\right)$ be admissible control-perturbation pairs from $U \times W$ such that

$$
u_{1}(t) \geq u_{2}(t) \text { and } w_{1}(t) \geq w_{2}(t) \text { a.e. } t \in[0, T],
$$


and let $y_{1}, y_{2} \in L^{2}(Q)$ be the corresponding generalized solutions to the parabolic system (1.1). Then we have the monotonicity relationship

$$
y_{1}(t, x) \geq y_{2}(t, x) \text { a.e. }(t, x) \in Q .
$$

Proof. Let us derive the formulated monotonicity property from the Maximum Principle for the classical solutions to the parabolic equations [8] by using an additional smooth approximation procedure. Denoting

$$
u(t):=u_{1}(t)-u_{2}(t), \quad w(t):=w_{1}(t)-w_{2}(t), \quad y(t, x):=y_{1}(t, x)-y_{2}(t, x),
$$

we conclude that $y \in L^{2}(Q)$ is a generalized solution to the parabolic system (1.1) corresponding to the nonnegative $L^{\infty}$-inputs

$$
u(t) \geq 0 \text { and } w(t) \geq 0 \text { a.e. } t \in[0, T] .
$$

Take further an arbitrary $C^{\infty}$-function $\rho: \mathbb{R} \rightarrow \mathbb{R}$ with the properties
(a) $\rho(r)=0$
if $|r| \geq 1$,
(b) $\rho(r) \geq 0$
if $|r| \leq 1$,
(c) $\int_{\mathbb{R}} \rho(r) d r=1$.

Then for any $v \in L^{2}[0, T]$ and $\varepsilon>0$ define

$$
v_{\varepsilon}(t):=\frac{1}{\varepsilon} \int_{\mathbb{R}} \rho\left(\frac{r-t}{\varepsilon}\right) v(r) d r
$$

which is a $C^{\infty}$-function such that $v_{\varepsilon} \rightarrow v$ strongly in $L^{2}[0, T]$ as $\varepsilon \downarrow 0$. Furthermore, $v_{\varepsilon}(t) \geq 0$ for all $t \in[0, T]$ whenever $v(t) \geq 0$ for a.e. $t \in[0, T]$.

Applying now the smooth approximation procedure (3.3) to the functions $u(t)$ and $w(t)$ from (3.2), we construct nonnegative functions $u_{\varepsilon}(t)$ and $w_{\varepsilon}(t)$ that generate, for each $\varepsilon>0$, the classical solution $y_{\varepsilon} \in C^{1,2}([0, T] \times \mathrm{cl} \Omega)$ to (1.1) satisfying

$$
y_{\varepsilon}(t, x) \geq 0 \text { for all }(t, x) \in \operatorname{cl} Q
$$

by the parabolic Maximum Principle. Passing to the limit in (3.4) as $\varepsilon \downarrow 0$ and taking into account that $u_{\varepsilon} \rightarrow u$ and $w_{\varepsilon} \rightarrow w$ strongly in $L^{2}\{0, T]$ which apply by (2.6) the strong convergence of $y_{\varepsilon} \rightarrow y$ in $L^{2}(Q)$, we arrive at (3.1) and complete the proof.

The monotonicity property established in Theorem 3.1 plays a crucial role in developing and justifying the design approach of this paper and in proving the most important results presented below. Let us next use this property and the transient convolution representation from [17], which also relies on the Maximum Principle for the parabolic dynamics, to select the worst perturbations in the feedback problem $(P)$.

In general, worst perturbations and optimal feedback controls are interrelated parts of saddle point solutions to minimax problems, which finding exactly is extremely difficult for dynamical systems of any type. We are going to simplify this task for the setting under consideration by taking into account the full sign symmetry of the initial data and constraints in $(P)$. To proceed efficiently in this way, we propose to split naturally the general case in $(P)$ into the two one-sided symmetric subcases:

$$
\begin{aligned}
& 0 \leq w(t) \leq \beta \text { and }-\alpha \leq u(t) \leq 0 \text { a.e. } t \in[0, T], \\
& -\beta \leq w(t) \leq 0 \text { and } 0 \leq u(t) \leq \alpha \text { a.e. } t \in[0, T] .
\end{aligned}
$$

This allows us to arrive at the following extreme value structure of the worst perturbations. 
Theorem 3.2 (worst perturbations). Under the standing assumptions, suppose that a feasible feedback $f \in \mathcal{F}$ is an odd and nonincreasing function on $\mathbb{R}$. Then the worst perturbations $w(t)$ providing the maximum value to the integral functional in (1.8) over all $w \in W$ from (1.4) are the extreme ones from the admissible area:

$$
w \equiv \beta \text { and } w \equiv-\beta \text {. }
$$

Proof. As mentioned, the admissible control and perturbation areas in (1.3) and (1.4) are fully symmetric with respect to the origin, and they both enter linearly to the linear parabolic system (1.1) with the homogeneous initial condition. The state constraint area in (1.5) is symmetric as well. To keep this symmetry in the feedback system (1.1) and (1.7) with the cost functional (1.8), we consider feedback laws in (1.7) given by odd functions $f=f(\xi)$, i.e., by those having the symmetry $f(-\xi)=-f(\xi)$ whenever $\xi \in \mathbb{R}$.

By [17, Theorem 5.1] the transients $\xi(t):=y\left(t, x_{0}\right)$ of $(1.1)$ generated by any admissible controls $u(t)$ and perturbations $w(t)$ have the following convolution representation

$$
\xi(t)=\varphi(t) * w(t)+\psi(t) * u(t):=\int_{0}^{t} \varphi(t-s) w(s) d s+\int_{0}^{t} \psi(t-s) u(s) d s
$$

for all $t \in[0, T]$, where both functions $\psi \in L^{\infty}[0, T]$ and $\varphi \in L^{\infty}[0, T]$ are positive on $[0, T]$ (due to the parabolic Maximum Principle) and are independent of $(u, w) \in U \times W$.

Fix a feedback function $f \in \mathcal{F}$ satisfying the aforementioned requirements. Consider for definiteness only the case (3.5); the other one in (3.6) is fully symmetric. Given a perturbation $w(t)$ under the constraints in (3.5), we have due to (1.7) that the corresponding Dirichlet boundary control $u(t)$ in (1.1) formed via this feedback law $f$ by

$$
u(t)=f\left(y\left(t, x_{0}\right)\right)=f(\xi(t)) \text { a.e. } t \in[0, T]
$$

and the cost functional (1.8) written as

$$
\int_{0}^{T}\left|f\left(y\left(t, x_{0}\right)\right)\right| d t=\int_{0}^{T}|u(t)| d t=-\int_{0}^{T} u(t) d t
$$

due to the control constraints in (3.5). By the convolution representation (3.8), the corresponding (one-sided) state constraints (1.5) imposed on $y\left(t, x_{0}\right)=\xi(t)$ have the form

$$
\int_{0}^{T} \varphi(t-s) w(s) d s+\int_{0}^{T} \psi(t-s) u(s) d s \leq \eta, \quad 0 \leq t \leq T .
$$

Since both functions $\varphi$ and $\psi$ are positive on $[0, T]$ (which is a crucial characteristic of the parabolic dynamics), it follows directly from (3.10) that the bigger magnitude of a perturbation is, the more control of the opposite sign should be applied to neutralize this perturbation in the sense of ensuring the required state performance (1.5), i.e., the fulfillment of (3.10) in the case under consideration. Taking into account the assumed nonincreasing property of $f(\xi)$, we get that the maximum value of the cost functional (3.9) over all the constraints in (3.5) and (3.10) corresponds to the maximal perturbation $w(t) \equiv \beta$ on $[0, T]$, which requires the maximal control response to keep (3.10) along the parabolic dynamics (1.1); the latter is due the positivity of $\varphi$ and $\psi$ in (3.10). This justifies the required conclusion for the extreme upper perturbation $w \equiv \beta$ in case (3.5). By symmetry we get the worst perturbation $w \equiv-\beta$ in the lower case (3.6) and thus complete the proof of the theorem.

Our next step is to consider problem $(P)$ under the worst perturbations (3.7). By the above discussions, it is sufficient to study only the one-sided case (3.5), since the other case 
(3.6) is completely symmetric. In case (3.5) the worst perturbation is detected as $w \equiv \beta$, and so the one-sided version of the initial problem $(P)$ under the fixed perturbation $w \equiv \beta$ reduces to the following feedback optimal control problem $(\widetilde{P})$ :

$$
\operatorname{minimize} \widetilde{J}(f):=-\int_{0}^{T} f\left(y\left(t, x_{0}\right)\right) d t \text { over } f \in \mathcal{F}
$$

subject to the closed-loop parabolic system

$$
\left\{\begin{array}{l}
\frac{\partial y}{\partial t}+A y=\beta \quad \text { a.e. }(t, x) \in Q, \\
y(0, x)=0, \quad x \in \Omega \\
y(t, x)=f\left(y\left(t, x_{0}\right)\right) d t \text { a.e. }(t, x) \in \Sigma
\end{array}\right.
$$

and the one-sided versions of the above control and state constraints.

Since problem $(\widetilde{P})$ does not involve any uncertainty and that boundary controls $u(t)$ are formed in the parabolic system (1.1) by the law $u(t)=f\left(y\left(t, x_{0}\right)\right)$ of $(1.7)$, we associate with $(\widetilde{P})$ the following open-loop optimal control problem $(\widetilde{P})$, which arises from $(\widetilde{P})$ by putting $u(t)$ instead of $f\left(y\left(t, x_{0}\right)\right)$ in both the cost functional and boundary conditions:

$$
\operatorname{minimize} \bar{J}(u):=-\int_{0}^{T} u(t) d t
$$

along the parabolic system

$$
\left\{\begin{array}{l}
\frac{\partial y}{\partial t}+A y=\beta \text { a.e. }(t, x) \in Q \\
y(0, x)=0, \quad x \in \Omega \\
y(t, x)=u(t) \text { a.e. }(t, x) \in \Sigma
\end{array}\right.
$$

with the fixed perturbation $w \equiv \beta$ and subject to the pointwise control and state constraints

$$
\begin{gathered}
u(\cdot) \in L^{\infty}[0, T] \text { with }-\alpha \leq u(t) \leq 0 \text { a.e. } t \in[0, T] \\
y\left(\cdot, x_{0}\right) \in L^{2}[0, T] \text { with } y\left(t, x_{0}\right) \leq \eta \text { a.e. } t \in[0, T]
\end{gathered}
$$

Note that problem $(\bar{P})$ is "broader" than $(\widetilde{P})$, since not every feasible control $u(t)$ can be represented as $u(t)=f\left(y\left(t, x_{0}\right)\right.$ with some $f \in \mathcal{F}$. On the other, in what follows we effciently compute a suboptimal feasible solution to problem $(\bar{P})$, which can be realized in form (1.7) with some feasible three-positional regulator $f \in \mathcal{F}$ satisfying all the imposed requirements. This justifies our approach to solve the feedback control problem $(P)$ by finding first a (sub)optimal open-loop control in response to the worst perturbations by solving problem $(\bar{P})$ and then realizing it via a feasible feedback regulator, which ensures the required state performance of the closed-loop control system for any admissible perturbations.

Focusing now on $(\bar{P})$, observe that it is an open-loop state-constrained Dirichlet boundary control problem, which was considered in $[14,19]$ in more generality; cf. also [3, 21]. In $[14,19]$ we obtained necessary optimality conditions for $(\bar{P})$ that involve the adjoint operator to the so-called Dirichlet map and Borel measures. These conditions are rather complicated and do not allow us to compute or even constructively estimate an optimal control. 
In this paper we develop, following $[13,16]$, another strategy to solve $(\widetilde{P})$ based first on $O D E$ approximations of the parabolic system (3.12) and then on subsequent penalty-type approximations of state constraints. To proceed, we use the spectral representation

$$
y\left(t, x_{0}\right)=\sum_{i=1}^{\infty} \mu_{i}\left(\frac{\beta}{\lambda_{i}}\left(e^{\lambda_{i} t}-1\right)+\left(c+\lambda_{i}\right) \int_{0}^{t} u(\theta) e^{\lambda_{i} \theta} d \theta\right) e^{-\lambda_{i} t} \phi_{i}\left(x_{0}\right)
$$

of solutions to the parabolic system (3.12) at $x=x_{0}$ implied by Proposition 2.3 (via straightforward integration), where the series in (3.15) converges strongly in $L^{2}[0, T]$. Now taking any natural $N=1,2, \ldots$, we replace series (3.15) by the finite $N$-sum

$$
y^{N}\left(t, x_{0}\right)=\sum_{i=1}^{N} \mu_{i}\left(\frac{\beta}{\lambda_{i}}\left(e^{\lambda_{i} t}-1\right)+\left(c+\lambda_{i}\right) \int_{0}^{t} u(\theta) e^{\lambda_{i} \theta} d \theta\right) e^{-\lambda_{i} t} \phi_{i}\left(x_{0}\right)
$$

for which $y^{N}\left(t, x_{0}\right) \rightarrow y\left(t, x_{0}\right)$ strongly in $L^{2}[0, T]$. Furthermore, it is easy to observe that $y^{N}\left(t, x_{0}\right)$ in (3.16) is represented as the sum

$$
y^{N}\left(t, x_{0}\right)=\sum_{i=1}^{N} y_{i}(t), \quad 0 \leq t \leq T,
$$

where each $y_{i}(t), i=1, \ldots, N$, satisfies the corresponding ordinary differential equation

$$
\dot{y_{i}}=-\lambda_{i} y_{i}+\mu_{i} \phi_{i}\left(x_{0}\right)\left(\beta+\left(c+\lambda_{i}\right) u(t)\right) \text { a.e. } t \in[0, T], y_{i}(0)=0 \text {. }
$$

Observe that the original feedback control problems $(P)$ as well as the open-loop optimal control problem $(\bar{P})$ of our current consideration are formulated on the fixed time interval $[0, T]$. In many applications (including those motivated this study) the time duration $T$ is large enough and can be conventionally taken as the infinite horizon, which allows us to involve $t \rightarrow \infty$ in our analysis. In the latter case the basic hypothesis $(H)$ in Section 2 and the inequalities in (2.3) ensure the dominance of the first terms in the exponential series and finite sums (3.15)-(3.17) as $t \rightarrow \infty$; see [18] for more discussions and numerical simulations.

Taking this into account, we pay a special attention to the case of $N=1$ in (3.16)(3.18) for determining an appropriate suboptimal control structure in problem $(\bar{P})$ for the parabolic equation (3.12), which provides an adequate approximation of the PDE dynamics on large time intervals. Then we optimize parameters of this suboptimal structure along both open-loop and closed-loop parabolic systems; see Sections 5 and 6 . Furthermore, the case of $t \rightarrow \infty$ is of crucial importance to establish verifiable conditions for robust stability of the highly nonlinear closed-loop parabolic control system studied in Section 7 via a variational approach. Overall the strategy developed in what follows eventually leads us to the efficient and easily implementable suboptimal control design of the hard-constrained parabolic systems under consideration.

In the next section we obtain the exact optimal solution to the open-loop control problem corresponding to the described ODE approximation as $N=1$ of the original parabolic system (3.12) subject to all the imposed pointwise control and state constraints on an arbitrary time interval $[0, T]$.

\section{Exact Solutions to Approximating ODE Control Problems}

According to the preceding discussions, we concentrate in this section on the study of the following open-loop optimal control problem $\left(\bar{P}_{1}\right)$ : minimize the cost functional (3.11) 
over admissible controls $u(t)$ satisfying the constraints in (3.13) and generating absolutely continuous trajectories $y:[0, T] \rightarrow \mathbb{R}$ of the ODE system

$$
\dot{y}=-\lambda_{1} y+\mu_{1} \phi_{1}\left(x_{0}\right)\left(\beta+\left(c+\lambda_{1}\right) u(t)\right) \text { a.e. } t \in[0, T], y(0)=0,
$$

subject to the pointwise state constraints

$$
y(t) \leq \eta \text { for all } t \in[0, T] .
$$

Observe that the presence of the state constraints (4.2) places problem $\left(\bar{P}_{1}\right)$ among the most challenging problems for ODE control. Available optimality conditions for such problems involve Borel measures that make them very difficult for implementations and applications; see, e.g., [5] and the references therein.

We develop a different approach to solve $\left(\bar{P}_{1}\right)$, which employs a penalty-type procedure to approximate state constraints, then deals with solving approximating problems in the absence of state constraints, and finally derives optimal solutions to the state-constrained problem $\left(\bar{P}_{1}\right)$ by passing to the limit from optimal solutions to the approximating problems.

This approach occurs to be highly efficient for the class of problems under consideration. It allows us to find exact optimal solutions to the approximating problems based on the Pontryagin maximum principle [20], which provides necessary and sufficient optimality conditions for these problems, and then to compute by passing to the limit the exact optimal control to the constrained problem $\left(\bar{P}_{1}\right)$. It surprisingly happens that the optimal control for the state-constraint problem enjoys a simpler structure in comparison with the unconstrained approximating problems, and that overall the state constraint (4.2) turns out to be a regularization factor in this setting.

Given $\varepsilon>0$, consider the approximating optimal control problem $\left(\bar{P}_{1 \varepsilon}\right)$ with no state constraints that is defined as follows:

$$
\operatorname{minimize} \bar{J}_{\varepsilon}(u):=\int_{0}^{T}\left(-u(t)+\frac{1}{\varepsilon}(\max \{0, y(t)-\eta\})^{2}\right) d t
$$

over controls $u(\cdot)$ satisfying (3.13) and the corresponding trajectories $y(\cdot)$ of the differential equation (4.1). It is easy to see that any control from (3.13) is feasible to $\left(\bar{P}_{1 \varepsilon}\right)$. The following theorem fully describes optimal controls to problems $\left(\bar{P}_{1 \varepsilon}\right)$ with $\varepsilon>0$ sufficiently small in the major cases needed for further applications.

Theorem 4.1 (optimal solutions to ODE approximation problems with no state constraints). Optimal controls $u_{\varepsilon}(t)$ to problems $\left(\bar{P}_{1 \varepsilon}\right)$ always exist and for every small $\varepsilon>0$ are determined as follows:

(i) Let either $\mu_{1} \phi_{1}\left(x_{0}\right) \beta \leq \lambda_{1} \eta$, or the conditions

$$
\mu_{1} \phi_{1}\left(x_{0}\right) \beta>\lambda_{1} \eta \text { and } \frac{\mu_{1} \phi_{1}\left(x_{0}\right) \beta}{\mu_{1} \phi_{1}\left(x_{0}\right) \beta-\lambda_{1} \eta} \geq e^{\lambda_{1} T}
$$

be satisfied. Then $u_{\varepsilon}(t) \equiv 0$ for a.e. $t \in[0, T]$.

(ii) Let $\mu_{1} \phi_{1}\left(x_{0}\right) \beta>\lambda_{1} \eta$ and both conditions

$$
\frac{\mu_{1} \phi_{1}\left(x_{0}\right) \beta}{\mu_{1} \phi_{1}\left(x_{0}\right) \beta-\lambda_{1} \eta}<e^{\lambda_{1} T}, \quad \mu_{1} \phi_{1}\left(x_{0}\right)\left(\beta-\alpha\left(c+\lambda_{1}\right)\right) \leq \lambda_{1} \eta
$$


be fulfilled. Then optimal controls $u_{\varepsilon}(t)$ to $\left(\bar{P}_{1 \varepsilon}\right)$ are computed by

$u_{\varepsilon}(t)= \begin{cases}0 & \text { if } t \in\left[0, \tau_{1 \varepsilon}\right) \cup\left(\tau_{2 \varepsilon}, T\right], \\ {\left[\frac{\lambda_{1} \eta}{\mu_{1} \phi_{1}\left(x_{0}\right)\left(c+\lambda_{1}\right)}-\frac{\beta}{c+\lambda_{1}}\right]+\frac{\lambda_{1}^{2} \varepsilon}{2 \mu_{1}^{2} \phi_{1}^{2}\left(x_{0}\right)\left(c+\lambda_{1}\right)^{2}}} & \text { if } t \in\left[\tau_{1 \varepsilon}, \tau_{2 \varepsilon}\right],\end{cases}$

where the switching times $0<\tau_{1 \varepsilon}<\tau_{2 \varepsilon}<T$ are given by

$$
\begin{gathered}
\tau_{1 \varepsilon}=\frac{1}{\lambda_{1}} \ln \left[1-\frac{\lambda_{1}}{\mu_{1} \phi_{1}\left(x_{0}\right) \beta}\left(\frac{\lambda_{1} \varepsilon}{2 \mu_{1} \phi_{1}\left(x_{0}\right)\left(c+\lambda_{1}\right)}+\eta\right)\right]^{-1} \\
\tau_{2 \varepsilon}=T+\frac{1}{\lambda_{1}} \ln \left[1-\lambda_{1} \sqrt{\frac{\varepsilon}{\mu_{1} \phi_{1}\left(x_{0}\right)\left(c+\lambda_{1}\right)\left(\mu_{1} \phi_{1}\left(x_{0}\right) \beta-\lambda_{1} \eta\right)}}\right] .
\end{gathered}
$$

Proof. The existence of optimal controls is ensured by the linearity of (4.1) and (3.11) with respect to the control variable and the convexity of the control region in (3.13); see [5].

To justify the precise formulas to compute optimal controls asserted in the theorem, consider first the case of

$$
\mu_{1} \phi_{1}\left(x_{0}\right) \beta \leq \lambda_{1} \eta
$$

in assertion (i). In this case the solution to the differential equation (4.1) corresponding to $u(t) \equiv 0$ on $[0, T]$ is given by

$$
y(t)=\frac{\mu_{1} \phi_{1}\left(x_{0}\right) \beta}{\lambda_{1}}\left(1-e^{-\lambda_{1} t}\right) \text { for all } t \in[0, T] .
$$

It is easy to see that (4.8) and (4.9) with $\lambda_{1}>0$ imply that the state constraint (4.2) holds for this $y(t)$, i.e. $u(t) \equiv 0$ gives the absolute minimum to the functional (4.3) over all $u(t) \leq 0$.

In case (4.4) of assertion (i) we again use the solution formula (4.9) for $y(t)$ corresponding to $u(t) \equiv 0$ on $[0, T]$ and confirm that

$$
y(t) \leq y(T) \leq \eta \text { for all } t \in[0, T]
$$

which ensures the global optimality of $u(t) \equiv 0$ for al $t \in[0, T]$ to $\left(\bar{P}_{1 \varepsilon}\right)$ and thus completes the proof of assertion (i).

To prove assertion (ii) of the theorem, we use the Pontryagin maximum principle [20], which provides necessary and sufficient conditions for optimal controls to the problems under consideration. According to this result, optimal controls $u_{\varepsilon}(t)$ to each problem $\left(\bar{P}_{1 \varepsilon}\right)$ are fully determined by the maximum condition

$$
\left(\mu_{1} \phi_{1}\left(x_{0}\right)\left(c+\lambda_{1}\right) p_{\varepsilon}(t)+1\right) u_{\varepsilon}(t)=\max _{-\alpha \leq u \leq 0}\left[\left(\mu_{1} \phi_{1}\left(x_{0}\right)\left(c+\lambda_{1}\right) p_{\varepsilon}(t)+1\right) u\right]
$$

for a.e. $t \in[0, T]$, where $p_{\varepsilon}(t)$ is an absolute continuous trajectory to the adjoint system

$$
\dot{p}_{\varepsilon}(t)=\lambda_{1} p_{\varepsilon}(t)+\frac{2}{\varepsilon} \max \left\{0, y_{\varepsilon}(t)-\eta\right\} \text { a.e. } t \in[0, T], \quad p_{\varepsilon}(T)=0
$$

with $y_{\varepsilon}(t)$ generated by $u_{\varepsilon}(t)$ via $(4.1)$. Thus each problem $\left(\bar{P}_{1 \varepsilon}\right)$ equivalently reduces to that of finding feasible controls $u_{\varepsilon}(t)$ satisfying the maximum condition (4.10) along with the corresponding trajectories $y_{\varepsilon}(t)$ and $p_{\varepsilon}(t)$ of the primal (4.1) and adjoint (4.11) systems. 
First observe that the maximum condition (4.10) uniquely determines the control $u_{\varepsilon}(t)$ at any point $t \in[0, T]$ where the expression

$$
\mu_{1} \phi_{1}\left(x_{0}\right)\left(c+\lambda_{1}\right) p_{\varepsilon}(t)+1
$$

is either positive (then $u_{\varepsilon}(t)=0$ ) or negative (then $u_{\varepsilon}(t)=-\alpha$ ). These are the socalled bang-bang points, where the optimal control takes one of the extreme values from the admissible control region $[-\alpha, 0]$. On the other hand, the maximum condition does not provide any information for points $t \in[0, T]$ at which the latter expression vanishes; these are the so-called singular points.

In what follows we are going to verify that for each $\varepsilon>0$ small enough the control $u_{\varepsilon}(t)$ asserted in (ii) with the switching times $\tau_{1 \varepsilon}$ and $\tau_{2 \varepsilon}$ from (4.6) and (4.7), respectively, is feasible to problem $\left(\bar{P}_{1 \varepsilon}\right)$ and satisfies the relationships of the Pontryagin maximum principle. This would justify the control optimality as discussed above.

It is easy to check that $u_{\varepsilon}(t) \leq 0$ for all $t \in[0, T]$ due to the assumed condition $\mu_{1} \phi_{1}\left(x_{0}\right) \beta>\lambda_{1} \eta$ and that $u_{\varepsilon}(t) \geq-\alpha$ for all $t \in[0, T]$ due to the second condition in (4.5). This confirms the feasibility of $u_{\varepsilon}(t)$ to $\left(\bar{P}_{1 \varepsilon}\right)$.

To prove the optimality of the given control $u_{\varepsilon}(t)$ with the switching times $\tau_{1 \varepsilon}$ and $\tau_{2 \varepsilon}$, we can directly substitute this control into (4.1) and (4.11), solve these equations for $y_{\varepsilon}(t)$ and $p_{\varepsilon}(t)$, and then check that the maximum condition (4.10) is fulfilled along the triple $\left(u_{\varepsilon}, y_{\varepsilon}, p_{\varepsilon}\right)$. Let us provide some calculations showing eventually how we come up to the precise formulas for computing the above optimal control $u_{\varepsilon}(t)$ in (ii).

One can easily verify that the switching times $\tau_{1 \varepsilon}$ and $\tau_{2 \varepsilon}$ calculated in (4.6) and (4.7), respectively, satisfy the inequalities

$$
0<\tau_{1 \varepsilon}<\tau_{2 \varepsilon}<T .
$$

Indeed, these inequalities directly follow from first two conditions assumed in (ii). Furthermore, let us demonstrate that the singularity condition

$$
\mu_{1} \phi_{1}\left(x_{0}\right)\left(c+\lambda_{1}\right) p_{\varepsilon}(t)+1 \equiv 0 \text { on }\left[\tau_{1 \varepsilon}, \tau_{2 \varepsilon}\right]
$$

is fulfilled along the adjoint trajectory $p_{\varepsilon}(t)$ generated by the control $u_{\varepsilon}(t)$ under consideration with the switching times $\tau_{1 \varepsilon}$ and $\tau_{2 \varepsilon}$ computed in (4.6) and (4.7). This would signify that the control $u_{\varepsilon}(t)$ is singular on the interval $\left[\tau_{1 \varepsilon}, \tau_{2 \varepsilon}\right]$. We show in fact that the singularity condition (4.13) allows us to find $u_{\varepsilon}(t)$ with the switching times $\tau_{1 \varepsilon}$ and $\tau_{2 \varepsilon}$ asserted in (ii).

To proceed, observe that the singularity condition (4.13) held on some interval $\left[\tau_{1 \varepsilon}, \tau_{2 \varepsilon}\right]$, not necessarily generated by (4.6) and (4.7), yields by differentiating identity (4.13) via the adjoint system (4.11) that

$$
0<\frac{\lambda_{1} \varepsilon}{2 \mu_{1} \phi_{1}\left(x_{0}\right)\left(c+\lambda_{1}\right)}=\max \left\{0, y_{\varepsilon}(t)-\eta\right\} \text { for all } t \in\left[\tau_{1 \varepsilon}, \tau_{2 \varepsilon}\right]
$$

and thus the corresponding singular arc $y_{\varepsilon}(\cdot)$ must be constant on $\left[\tau_{1 \varepsilon}, \tau_{2 \varepsilon}\right]$ and equal to

$$
y_{\varepsilon}(t)=\eta+\frac{\lambda_{1} \varepsilon}{2 \mu_{1} \phi_{1}\left(x_{0}\right)\left(c+\lambda_{1}\right)}, \quad t \in\left[\tau_{1 \varepsilon}, \tau_{2 \varepsilon}\right] .
$$

Combining (4.14) with (4.1), we inevitably arrive at the intermediate value of $u_{\varepsilon}(t)$-within the admissible region $[-\alpha, 0]-$ on the singularity interval $\left[\tau_{1 \varepsilon}, \tau_{2 \varepsilon}\right]$ from (4.13). To justify 
the exact formulas for $\tau_{1 \varepsilon}$ and $\tau_{2 \varepsilon}$ asserted in (4.6) and (4.7), let us compute the switching times from (4.14) providing that the control $u_{\varepsilon}(t)$ is given by the expression in (ii) with some numbers $\tau_{1 \varepsilon}$ and $\tau_{2 \varepsilon}$ from (4.13). Indeed, we easily get from (4.14) and formula (4.9) for $y_{\varepsilon}(t)$ on $\left[0, \tau_{1 \varepsilon}\right]$ generated by $u_{\varepsilon}(t) \equiv 0$ that $\tau_{1 \varepsilon}$ must satisfy the equation

$$
\frac{\lambda_{1} \varepsilon}{2 \mu_{1} \phi_{1}\left(x_{0}\right)\left(c+\lambda_{1}\right)}+\eta=\frac{\mu_{1} \phi_{1}\left(x_{0}\right) \beta}{\lambda_{1}}\left(1-e^{-\lambda_{1} \tau_{1 \varepsilon}}\right)
$$

which elementary leads to formula (4.6) for computing the asserted time $\tau_{1 \varepsilon}$.

Next take the control $u_{\varepsilon}(t)$ from (ii) with $\tau_{1 \varepsilon}$ computed by (4.6) and with some (not precisely known so far) switching time $\tau_{2 E}$ satisfying the singularity condition (4.13) and find $\tau_{2 \varepsilon}$ explicitly from the latter condition. Using $y_{\varepsilon}(t)$ on $\left[\tau_{1 \varepsilon}, \tau_{2 \varepsilon}\right]$ from (4.14), we compute $y_{\varepsilon}(t)$ on $\left[\tau_{2 \varepsilon}, T\right]$ with $\tau_{1 \varepsilon}$ from (4.14) and an unknown time $\tau_{2 \varepsilon}$ by integrating the following system:

$$
\dot{y}_{\varepsilon}=-\lambda_{1} y_{\varepsilon}+\mu_{1} \phi_{1}\left(x_{0}\right) \beta \text { on }\left[\tau_{2 \varepsilon}, T\right], \quad y_{\varepsilon}\left(\tau_{2 \varepsilon}\right)=\frac{2 \eta \mu_{1} \phi_{1}\left(x_{0}\right)\left(c+\lambda_{1}\right)+\lambda_{1} \varepsilon}{2 \mu_{1} \phi_{1}\left(x_{0}\right)\left(c+\lambda_{1}\right)} .
$$

The solution of the latter initial value problem is

$$
y_{\varepsilon}(t)=\frac{\mu_{1} \phi_{1}\left(x_{0}\right) \beta}{\lambda_{1}}+e^{\lambda_{1}\left(\tau_{2 \varepsilon}-t\right)}\left(\frac{2 \eta \mu_{1} \phi_{1}\left(x_{0}\right)\left(c+\lambda_{1}\right)+\lambda_{1} \varepsilon}{2 \mu_{1} \phi_{1}\left(x_{0}\right)\left(c+\lambda_{1}\right)}-\frac{\mu_{1} \phi_{1}\left(x_{0}\right) \beta}{\lambda_{1}}\right)
$$

on the time interval $\left[\tau_{2 \varepsilon}, T\right]$. Substituting (4.15) into the adjoint system (4.11), find the corresponding adjoint trajectory $p_{\varepsilon}(t)$ on $\left[\tau_{2 \varepsilon}, T\right]$. Taking into account that

$$
p_{\varepsilon}\left(\tau_{2 \varepsilon}\right)=\frac{1}{\mu_{1} \phi_{1}\left(x_{0}\right)\left(c+\lambda_{1}\right)},
$$

we arrive at the following transcendental equation for $\tau_{2 \varepsilon}$ :

$$
\begin{aligned}
& \frac{\lambda_{1} \varepsilon}{2 \mu_{1} \phi_{1}\left(x_{0}\right)\left(c+\lambda_{1}\right)}=\frac{\mu_{1} \phi_{1}\left(x_{0}\right) \beta}{2 \lambda_{1}}-\frac{\mu_{1} \phi_{1}\left(x_{0}\right) \beta}{2 \lambda_{1}} e^{\lambda_{1}\left(\tau_{2 \varepsilon}-T\right)} \\
& -\left(\frac{2 \eta \mu_{1} \phi_{1}\left(x_{0}\right)\left(c+\lambda_{1}\right)+\lambda_{1} \varepsilon}{4 \mu_{1} \phi_{1}\left(x_{0}\right)\left(c+\lambda_{1}\right)}-\frac{\mu_{1} \phi_{1}\left(x_{0}\right) \beta}{\lambda_{1}}\right) e^{2 \lambda_{1}\left(\tau_{2 \varepsilon}-T\right)} \\
& +\eta e^{\lambda_{1}\left(\tau_{2 \varepsilon}-T\right)}+\frac{\lambda_{1} \varepsilon}{4 \mu_{1} \phi_{1}\left(x_{0}\right)\left(c+\lambda_{1}\right)}-\frac{\eta}{2}
\end{aligned}
$$

which is actually the quadratic equation with respect to the variable $z:=e^{\lambda_{1} \tau_{2 \varepsilon}}$. The latter equation has the only solution $\tau_{2 \varepsilon}$ belonging to the required interval $\left(\tau_{1 \varepsilon}, T\right)$-it is given by the explicit formula (4.7); the second solutions gives $\tau_{2 \varepsilon}>T$ for all $\varepsilon>0$, which is not acceptable. This completes the proof of the theorem.

Theorem 4.1 establishes that, in the most interesting case of assertion (ii), optimal controls to the approximating problems $\left(\bar{P}_{1 \varepsilon}\right)$ are piecewise constant functions consisting of three parts: they start for all $\varepsilon>0$ from the upper extreme value $u=0$ (minimal resource), then switch to the intermediate positions (depending on $\varepsilon$ ) from the admissible control region, and finally come back to the same extreme value $u=0$. Note that the other extreme value $u=-\alpha$ (maximum resource) is never used in the optimal control designed in (ii), and that the intermediate control values in (ii) are manifestations of singular modes. 
Observe also that we did not consider in Theorem 4.1 one more-remaining-possibility of parameter combinations for $\left(\bar{P}_{1 \varepsilon}\right)$, namely:

$$
\left\{\begin{array}{l}
\mu_{1} \phi_{1}\left(x_{0}\right) \beta>\lambda_{1} \eta, \frac{\mu_{1} \phi_{1}\left(x_{0}\right) \beta}{\mu_{1} \phi_{1}\left(x_{0}\right) \beta-\lambda_{1} \eta}<e^{\lambda_{1} T}, \\
\mu_{1} \phi_{1}\left(x_{0}\right)\left(\beta-\alpha\left(c+\lambda_{1}\right)\right)>\lambda_{1} \eta .
\end{array}\right.
$$

It follows from the proof of Theorem 4.1 that this case does not allow any singular mode of optimal control, which cannot also take the lower recourse $u \equiv 0$ on the whole interval $[0, T]$. According to the Pontryagin maximum principle, optimal controls $u_{\varepsilon}(t)$ to $\left(\bar{P}_{1 \varepsilon}\right)$ in this case must be bang-bang on the whole $[0, T]$ changing their positions from 0 to $-\alpha$. A detailed study of this case is not of our interest, since the parameter combinations in (4.16) exclude in fact the fulfillment of the state constraint (4.2), i.e., there are no feasible controls to our main problem $\left(\bar{P}_{1}\right)$ studied in this section; see the next theorem for the full description of feasibility/controllability and optimality in the state-constrained problem $\left(\bar{P}_{1}\right)$.

Theorem 4.2 (full description of controllability and optimality in the stateconstrained ODE control problem). The state constrained problem $\left(\bar{P}_{1}\right)$ is controllable, i.e., there is a feasible control to $\left(\bar{P}_{1}\right)$, if and only if one of the following cases holds:

(a) $\mu_{1} \phi_{1}\left(x_{0}\right) \beta \leq \lambda_{1} \eta$

(b) both conditions

$$
\mu_{1} \dot{\phi_{1}}\left(x_{0}\right) \beta>\lambda_{1} \eta, \quad \frac{\mu_{1} \phi_{1}\left(x_{0}\right) \beta}{\mu_{1} \phi_{1}\left(x_{0}\right) \beta-\lambda_{1} \eta} \geq e^{\lambda_{1} T}
$$

are satisfied simultaneously;

(c) all the three conditions

$$
\mu_{1} \phi_{1}\left(x_{0}\right) \beta>\lambda_{1} \eta, \quad \frac{\mu_{1} \phi_{1}\left(x_{0}\right) \beta}{\mu_{1} \phi_{1}\left(x_{0}\right) \beta-\lambda_{1} \eta}<e^{\lambda_{1} T}, \quad \mu_{1} \phi_{1}\left(x_{0}\right)\left(\beta-\alpha\left(c+\lambda_{1}\right)\right) \leq \lambda_{1} \eta
$$

are fulfilled simultaneously.

Furthermore, the constant function

$$
\bar{u}(t) \equiv 0 \text { on }[0, T]
$$

is an optimal control to problem $\left(\bar{P}_{1}\right)$ in both cases (a) and (b). In case (c) an optimal control to $\left(\bar{P}_{1}\right)$ is given by the two-positional piecewise constant function

$$
\bar{u}(t)= \begin{cases}0 & \text { if } t \in[0, \tau), \\ v:=\frac{\lambda_{1} \eta-\mu_{1} \phi_{1}\left(x_{0}\right) \beta}{\mu_{1} \phi_{1}\left(x_{0}\right)\left(c+\lambda_{1}\right)} & \text { if } t \in[\tau, T],\end{cases}
$$

where the switching time $\tau$ is computed by

$$
0<\tau:=\frac{1}{\lambda_{1}} \ln \frac{\mu_{1} \phi_{1}\left(x_{0}\right) \beta}{\mu_{1} \phi_{1}\left(x_{0}\right) \beta-\lambda_{1} \eta}<T .
$$

Proof. The feasibility of the trivial control (4.17) to problem $\left(\bar{P}_{1}\right)$ in both cases (a) and (b) is proved in Theorem 4.1, where it is shown that the corresponding trajectory $y(t)$ to (4.1) satisfies the state constraint (4.2) under the assumptions imposed in these cases. 
The optimality of (4.17) to $\left(\bar{P}_{1}\right)$ obviously follows from the structure of the cost functional (3.11) in $\left(\bar{P}_{1}\right)$. To verify the feasibility of control $(4.18)$ to $\left(\bar{P}_{1}\right)$ in case (c), we observe that $0<\tau<T$ in (4.19) under the first two conditions imposed in (c), that $-\alpha<\bar{u}(t) \leq 0$ on $[0, T]$ due to the first and third conditions therein, and that the state constraint (4.2) holds for the corresponding trajectory $y(t)$ to $(4.1)$ as one can easily verify by substituting (4.18) into (4.1) and elementary integration.

To justify that the union of the conditions in (a)-(c) is not only sufficient but also necessary for the existence of feasible controls to $\left(\bar{P}_{1}\right)$, i.e., it fully describes the controllability of $\left(\bar{P}_{1}\right)$, we now show that in the remaining case (4.16) there is no control from the admissible region (3.13) that generates a trajectory to (4.1) satisfying the state constraint (4.2). By the monotonicity property of Theorem 3.1, it is sufficient to check that the trajectory $y(t)$ generated by the control $u(t) \equiv-\alpha$ with the maximum resource violates the state constraint (4.2). To proceed, we substitute $u(t) \equiv-\alpha$ into (4.1) and get by integration of (4.1) that

$$
y(t)=\frac{\mu_{1} \phi_{1}\left(x_{0}\right)\left(\beta-\left(c+\lambda_{1}\right) \alpha\right)}{\lambda_{1}}\left(1-e^{-\lambda_{1} t}\right) \text { on } t \in[0, T]
$$

This immediately implies by the lower condition in (4.16) that $y(T)>\eta$, which shows that problem $\left(\bar{P}_{1}\right)$ is not controllable in case (4.16).

To complete the proof of the theorem, it remains to demonstrate that the feasible control $\bar{u}(t)$ from (4.18) with the switching time $\tau$ computed in (4.19) is optimal to problem $\left(\bar{P}_{1}\right)$ in case (c). Indeed, it easy to confirm from the formulas (4.6) for $\tau_{1 \varepsilon},(4.7)$ for $\tau_{2 \varepsilon}$, and (4.19) for $\tau$ that we have

$$
\tau_{1 \varepsilon} \downarrow \tau \text { and } \tau_{2 \varepsilon} \uparrow T \text { as } \varepsilon \downarrow 0 \text {. }
$$

Furthermore, it obvious that the intermediate positions of the controls $u_{\varepsilon}(t)$ in Theorem 4.1(ii) converge to $v \in[-\alpha, 0)$ in (4.18) as $\varepsilon \downarrow 0$. Thus

$$
\int_{0}^{T} u_{\varepsilon}(t) d t \rightarrow \int_{0}^{T} \bar{u}(t) d t \text { as } \varepsilon \downarrow 0 .
$$

The latter immediately implies the optimality of $\bar{u}(t)$ to $\left(\bar{P}_{1}\right)$ by the optimality of $u_{\varepsilon}(t)$ to $\left(\bar{P}_{1 \varepsilon}\right)$ established in Theorem 4.1.

We can see, by comparison the optimal control $\bar{u}(t)$ from (4.18) derived for the stateconstrained problem $\left(\bar{P}_{1}\right)$ in Theorem 4.2 with the ones $u_{\varepsilon}(t)$ derived in Theorem 4.1(ii) for the approximating problems $\left(\bar{P}_{1 \varepsilon}\right)$ with no state constraints, that the two-part piecewise constant optimal control to the state-constrained problem does not depend on any Borel measure in the adjoint system and turns out to be even simpler than those to $\left(\bar{P}_{1 \varepsilon}\right)$ consisting of three parts. It is a surprising conclusion that fully relies on the specific features of approximating ODE systems to the parabolic dynamics and signifies a regularization role of state constraints for the optimal control problems under consideration.

\section{Open-Loop Optimal Control of the Parabolic System un- der Worst Perturbations}

The results on controllability and on computing the optimal control to problem $\left(\bar{P}_{1}\right)$ derived in Theorem 4.2 can be treated as the first-order approximation to the general case of problem 
$(\bar{P})$ for the parabolic system under consideration. This approximation is fairly adequate to the general setting on a long time interval due to the basic assumption $(\mathrm{H})$ on the positivity of the first eigenvalue, which ensures the dominance of the first term in the solution representation for the parabolic system; see Section 2 for more details and discussions.

In this section we address the open-loop optimal control problem $(\bar{P})$ involving the parabolic dynamic and pointwise state constraints formulated in Section 3 while confine our study to optimizing the two-positional control structure well justified in Section 4. It means that we now consider the following dynamic optimization problem $(\widehat{P})$ depending in fact on two control parameters:

$$
\operatorname{minimize} J(v, \tau):=-\int_{0}^{T} u(t) d t
$$

over admissible Dirichlet boundary controls of the form

$$
u(t)=\left\{\begin{aligned}
0 & \text { if } t \in[0, \tau] \\
-v & \text { if } t \in(\tau, T]
\end{aligned}\right.
$$

subject to the constraints on control recourses $v$ and switching times $\tau$ given by

$$
0 \leq v \leq \alpha, \quad 0 \leq \tau \leq T
$$

and the pointwise state constraint (3.14) along the corresponding trajectories of the parabolic system (3.12). As seen in Section 4, the intermediate position $v$ in (5.2) is a characteristic feature of the singular control mode that leads us to the simple (while rigorously justified) suboptimal control structure in (5.2), which is significantly more convenient for further applications and implementations in comparison with those arising from the complicated and not efficiently verifiable necessary optimality conditions established in $[3,14,19,21]$ that involve, in particular, Borel measures.

In what follows we find an exact optimal solution to problem $(\widehat{P})$, which therefore provides a suboptimal solution to the general open-loop control problem $(\bar{P})$ formulated in Section 3, at least for all $T$ sufficiently large. Furthermore, we derive-in the process of optimization-constructive and simple conditions on the given parameters of the original parabolic system and imposed constraints that ensure the controllability in $(\hat{P})$, i.e., the existence of feasible controls to this problem and hence to problem $(\bar{P})$. The sufficient conditions obtained in this way turn out to be also necessary for controllability of $(\widehat{P})$ on any time interval $[0, T]$, i.e., when the problem is considered on the infinite horizon $[0, \infty)$.

To proceed, we define an aggregate spectral parameter of the strongly uniformly elliptic operator $A$ from (1.2) by

$$
\gamma:=\sum_{i=1}^{\infty} \frac{\mu_{i} \phi_{i}\left(x_{0}\right)}{\lambda_{i}}
$$

The next theorem contains controllability conditions for $(\widehat{P})$ and provides computing optimal control parameters for this problem.

Theorem 5.1 (controllability and optimal parameters of open-loop suboptimal control structure for the constrained parabolic system). Under the standing assumptions made, the following hold: 
(i) The parabolic system (3.12) is controllable on $[0, T]$ by piecewise constant Dirichlet boundary control functions (5.2) with parameters $(v, \tau)$ from $(5.3)$ subject to the state constraint (3.14) if one has the conditions

$$
0<\gamma \beta-\eta \leq \min \left\{\alpha(1+c \gamma), \frac{\beta(1+c \gamma)}{c+\lambda_{1}}\right\}
$$

Moreover, conditions (5.5) are necessary and sufficient for the controllability of (3.12) by (5.2) subject to (5.3) and (3.14) with any $T>0$, i.e., on the infinite horizon $[0, \infty)$.

(ii) Imposing conditions (5.5), consider the transcendental equation

$$
\sum_{i=1}^{\infty} \frac{\mu_{i} \phi_{i}\left(x_{0}\right)}{\lambda_{i}} e^{-\lambda_{i} T}\left[\left(c+\lambda_{i}\right)(\gamma \beta-\eta) e^{\lambda_{i} \tau}-\beta(1+c \gamma)\right]=0
$$

which has the unique solution $\tau=\tau(T) \in(0, T)$ for all $T$ sufficiently large. Then the boundary control $u(t)$ from (5.2) with the recourse

$$
v:=\frac{\gamma \beta-\eta}{1+c \gamma}
$$

is feasible to $(\widehat{P})$ for all positive switching times $\tau \leq \tau(T)$ being optimal to this problem among those with resource (5.7) when $\tau=\tau(T)$. Furthermore, there is the limit $\tau(T) \downarrow \bar{\tau}$ as $T \rightarrow \infty$, where the asymptotically optimal switching time $\bar{\tau}$ is computed by

$$
\bar{\tau}:=\frac{1}{\lambda_{1}} \ln \frac{\beta(1+c \gamma)}{\left(c+\lambda_{1}\right)(\gamma \beta-\eta)}
$$

and turns out to be maximal among all the switching times $\tau \geq 0$ in (5.2) ensuring the fulfilment of the state constraint (3.14) whenever $t \geq 0-i . e .$, on the infinite horizon $[0, \infty)-$ along the corresponding solutions $y\left(t, x_{0}\right)$ to the parabolic system (3.12) generated by the Dirichlet boundary controls (5.2) with the recourse $v \in(0, \alpha]$ from $(5.7)$.

Proof. Let $y(t, x)$ be the trajectory of the parabolic system (3.12) generated by some piecewise continuous Dirichlet boundary control $u(t)$ from (5.2) with parameters $(v, \tau)$ satisfying (5.3). For convenience we denote

$$
y(t):=y\left(t, x_{0}\right) \text { on }[0, \tau] \text { and } y(t ; \tau):=y\left(t, x_{0}\right) \text { on }[\tau, T] \text {. }
$$

By the spectral representation of Proposition 2.3 we have

$$
\begin{gathered}
y(t)=\beta\left(\gamma-\sum_{i=1}^{\infty} \frac{\mu_{i} \phi_{i}\left(x_{0}\right)}{\lambda_{i}} e^{-\lambda_{i} t}\right) \text { for } t \in[0, \tau] \\
y(t ; \tau)=\gamma \beta-(1+c \gamma) v+\sum_{i=1}^{\infty} \frac{\mu_{i} \phi_{i}\left(x_{0}\right)}{\lambda_{i}} e^{-\lambda_{i} t}\left[\left(c+\lambda_{i}\right) v e^{\lambda_{i} \tau}-\beta\right]
\end{gathered}
$$

for $t \in[\tau, T]$ with the same value $y(\tau)=y(\tau ; \tau)$ at the common point of (5.9) and (5.10).

Let $t=T_{0}>0$ be a solution to the equation $y(t)=\eta$, which exists by $\gamma \beta-\eta>0$ and is unique due to (5.9) under the standing assumptions of Section 2. When $T \leq T_{0}$, the control 
$u(t) \equiv 0$ on $[0, T]$ is obviously feasible and hence optimal to both problems $(\widehat{P})$ and $(\bar{P})$. In what follows we consider the case when the final time $T>T_{0}$ is sufficiently large. Since

$$
y(t ; \tau) \rightarrow \gamma \beta-(1+c \gamma) v \text { as } t \rightarrow \infty
$$

every control from (5.2) with any $\tau \geq 0$ stabilizes the corresponding transient (5.10) at the upper boundary $y=\eta$ of the state constraint region exponentially approaching the stabilized level (5.11) as $t \rightarrow \infty$. Selecting the control recourse $v$ by (5.7) and using the controllability conditions

$$
0<\gamma \beta-\eta \leq \alpha(1+c \gamma)
$$

from (5.5), we conclude that every control (5.2) is admissible by (5.3). It immediately follows from (5.11) that otherwise there is no control of type (5.2) that is feasible by keeping the pointwise state constraint (3.14) along the parabolic system (3.12) for $T$ sufficiently large, i.e., there is definitely no controllability in the problem $(\widehat{P})$ under consideration. Furthermore, the controllability may be violated even under conditions (5.12)-in the sense that the pointwise state constraint (3.14) is not preserved whenever $t \in[0, T]$-if the switching time $\tau$ is not properly selected in (5.2). Let us now demonstrate that the choice of $\tau$ as the (unique) solution to the transcendental equation (5.6) ensures-under all the conditions in (5.5)-first the controllability in problem $(\widehat{P})$ and, moreover, the optimality of the feasible control (5.2) with parameters (5.6) and (5.7) to this problem.

Indeed, consider all $\tau$ from (5.3) with $v$ computed in (5.7) such that $y(t ; \tau) \leq \eta$ whenever $t \in[0, T]$ for the transient $(5.10)$ generated by $u(t)$ from (5.2) corresponding to this pair $(v, \tau)$. Due to the monotonicity property of $y(t ; \tau)$ with respect to $\tau$-this follows from Theorem 3.1, the maximal among all such $\tau$ is the one satisfying the equation

$$
y(T, \tau(T))=\eta
$$

which is exactly that of (5.6). It is easy to observe from the explicit expression (5.10) for $y(t ; \tau)$ that the transcendental equation (5.6) has the unique solution $\tau(T)<T$ for all $T>T_{0}$. Moreover, we can check that $\tau(T)>0$ if

$$
\gamma \beta-\eta \leq \frac{\beta(1+c \gamma)}{c+\lambda_{1}}
$$

which finally ensures the controllability in $(\widehat{P})$ by controls $(5.2)$ on $[0, T]$ under the validity of all the conditions in (5.5). Further, it follows from structure (5.1) of the cost functional in $(\widehat{P})$ that the control $u(t)$ from (5.2) and (5.7) with the maximal $\tau=\tau(T)$ keeping the state constraint (3.14) is indeed optimal to $(\widehat{P})$ among those with resource (5.7).

It remains to consider the behavior of problem $(\widehat{P})$ when $T \rightarrow \infty$. It follows from the Maximum Principle for parabolic equations (similarly to the proof of Theorem 3.1) that the optimal switching time function $\tau(T)$ is strictly decreasing in $T$ being obviously bounded from below. Thus $\tau(T)$ converges as $T \rightarrow \infty$, and its limit $\bar{\tau}$ reduces to that computed in (5.8) due to the eigenvalue properties (2.3), which reflect the first eigenvalue dominance.

Finally, we observe directly from (5.10) that the control $u(t)$ from (5.2) with the resource $v$ computed in (5.7) and the switching time $\tau=\bar{\tau}$ computed in (5.8) preserves-with the strict inequality - the state constraint (3.14) for the corresponding transient in (5.9) and (5.10) whenever $t \geq 0$, i.e., it is feasible to problem $(\widehat{P})$ on the infinite horizon $[0, \infty)$. Furthermore, $\bar{\tau}$ is the maximal $\tau$ in (5.2) satisfying this property. The latter can be shown by 
applying the Fermat stationary rule to $(5.10)$ on the open interval $(\tau, \infty)$ via differentiation of $y(t ; \tau)$ in $t$ and checking that the maximum of $y(t ; \tau)$ over $(\tau, \infty)$ is bigger than $\eta$ whenever $\tau>\bar{\tau}$. Since $\bar{\tau}=0$ in (5.8) when

$$
\gamma \beta-\eta=\frac{\beta(1+c \gamma)}{c+\lambda_{1}}
$$

we thus confirm that the conditions in (5.5) are necessary and sufficient for the controllability in $(\widehat{P})$ on the infinite horizon $[0, \infty)$ and that the control $u(t)$ from (5.2) with $v$ from (5.7) and $\tau=\bar{\tau}$ is optimal to $(\widehat{P})$ when $T=\infty$. This completes the proof of the theorem.

Observe that the asymptotically optimal switching time $\bar{\tau}$ in (5.8) can be computed directly from the condition of vanishing the first term in the series of (5.10), i.e., from

$$
\left(c+\lambda_{1}\right) v e^{\lambda_{1} \tau}-\beta=0
$$

with $v$ given in (5.7). This justifies the simple and convenient first term rule to deal with the parabolic dynamics under the basic assumption $(\mathrm{H})$ as $t \rightarrow \infty$; see also Section 7 below.

The results derived in Theorem 5.1 particularly demonstrate that the passage to the infinite horizon allows us to significantly simplify optimal solutions to the open-loop control problems under consideration and to arrive at the convenient analytic formulas for computing their optimal parameters. The discovered phenomenon reveals a certain turnpike property, which is a characteristic feature of such state-constrained control problems governed by the parabolic dynamics.

\section{Feedback Suboptimal Control of the Parabolic System}

In the previous section we computed optimal parameters of the suboptimal two-positional control structure for the open-loop control problem $(\bar{P})$, which describes the best possible reaction of the control system to keep the required state constraints under the realization of the upper case of the worst perturbations $w(t) \equiv \beta$ on $[0, T]$. Due to the full symmetry of the initial problem $(P)$ discussed above, the lower case $w(t) \equiv-\beta$ of the worst perturbations on $[0, T]$ can be considered similarly by using open-loop Dirichlet boundary controls

$$
u(t)= \begin{cases}0 & \text { if } t \in[0, \tau] \\ v \in[0, \alpha] & \text { if } t \in(\tau, T]\end{cases}
$$

for the linear parabolic system

$$
\left\{\begin{array}{l}
\frac{\partial y}{\partial t}+A y=-\beta \quad \text { a.e. }(t, x) \in Q \\
y(0, x)=0, \quad x \in \Omega \\
y(t, x)=u(t) \text { a.e. }(t, x) \in \Sigma
\end{array}\right.
$$

subject to the pointwise state constraint

$$
y\left(\cdot, x_{0}\right) \in L^{2}[0, T] \text { with } y\left(t, x_{0}\right) \geq-\eta \text { a.e. } t \in[0, T] .
$$

Then, taking into account the sign changes in (6.1)-(6.3), the controllability conditions and formulas for computing the optimal control parameters in problem of

$$
\text { minimize } \int_{0}^{T} u(t) d t \quad \text { over constraints }(6.1)-(6.3)
$$


are exactly the same as in Theorem 5.1 for problem $(\widehat{P})$ formulated in Section 5 .

Now we are going to employ these results to construct a suboptimal feedback regulator for the original minimax feedback control problem $(P)$ described in Section 1. Recall that the purpose of feedback controls in $(P)$ is to keep transients within the given state constraint region (1.5) for all uncertain perturbations $w \in W$ from (1.4) subject to the imposed constrains on controls in such a way that the cost functional (1.8) is minimized under the realization of the worst perturbations. The results obtained above for computing (sub)optimal open-loop controls in the case of the worst perturbations allow us to justify the following suboptimal structure $f=f(\xi)$ of feedback controls (1.7) acting in the Dirichlet boundary conditions of the parabolic system (1.1):

$$
f(\xi)=\left\{\begin{array}{cl}
-v & \text { if } \xi \geq \sigma \\
0 & \text { if }-\sigma<\xi<\sigma \\
v & \text { if } \xi \leq-\sigma
\end{array}\right.
$$

describing a three-positional feedback regulator with the "dead region" $(-\sigma, \sigma)$. Observe that the feedback law $f(\xi)$ in $(6.5)$ is given by an odd and nonincreasing function satisfying the requirements of Theorem 3.2 .

By the structure of the boundary control dynamics in (1.1) with the feedback law of (1.7) and (6.5), the closed-loop control system under consideration is functioning as follows:

Whatever perturbation $w=w(t)$ is realized in the dynamical system, the control reacts only to the current state position $\xi=y\left(t, x_{0}\right)$ under observation, applying its zero recourse $u=0$ if $y\left(t, x_{0}\right)$ is within the dead region $-\sigma<\xi<\sigma$. When the state position $\xi$ reaches the upper bound $\sigma$ of the dead region, the control applies its lower recourse $u=-v$ in (6.5) and keeps it all the time while the state position $\xi$ exceeds the upper admissible level $\xi=\sigma$; then it applies again its zero recourse $u=0$ whenever the state position comes back to the dead region. The control system behavior is fully symmetric when the state position $\xi$ reaches (and then exceeds from below) the lower bound $\xi=-\sigma$ of the dead region.

The feedback control synthesis design-in the minimax sense of problem $(P)$-reduces now to determining appropriate parameters $(v, \sigma)$ in $(6.5)$ such that the resulting closedloop control system keeps the state position $\xi=y\left(t, x_{0}\right)$ under observation-starting with the initial equilibrium state $y(0, x) \equiv 0$ on $\Omega$-within the admissible state constraint area (1.5) whatever uncertain perturbation $w \in W$ is realized and then ensures the minimum value of the cost functional (1.8) under the realization of the worst perturbations.

According to the results obtained above for the open-loop control problems $(\widehat{P})$ and $(6.4)$, we can employ the control recourse $v$ in (6.5) computed by (5.7) for all $T$ sufficiently large, which is in fact necessary for stabilizing transients at the boundary of the state constraint region as $T \rightarrow \infty$ by using feedback regulators of the suboptimal three-positional structure (6.5). Note that the value $v$ in (5.7) is not the maximal available control recourse from the admissible region $[0, \alpha]$-besides the extreme case in the controllability conditions in (5.5)while, being a characteristics of the singular control mode, it ensures the optimal control response, with respect to minimizing the cost functional (1.8), to the worst perturbations. Our intention now is to find verifiable conditions on the remaining parameter $\sigma>0$ in (6.5) such that the resulting closed-loop control system meets the controllability/feasibility and minimax optimality requirements formulated above.

To give satisfactory calculations of the dead region parameter $\sigma$ in the next theorem, consider the functioning mode of the three-positional regulator (1.7) corresponding to the initial state position $y(0, x) \equiv 0$ on $\Omega$. Under this condition we answer both controllability 
and optimality questions formulated above, providing in fact the exact calculation of the optimal value $\sigma(T)$ on $[0, T]$ and fully describe its limiting/asymptotic behavior as $T \rightarrow \infty$. The latter relates to the counterpart of problem $(P)$ on the infinite horizon.

Theorem 6.1 (feasible and optimal parameters of the three-positional regulator in the minimax feedback control problem for the parabolic system). Consider the minimax feedback control problem $(P)$ under the standing assumptions made in the mode of functioning with the initial state position $y(0, x) \equiv 0$ on $\Omega$. Let the feedback boundary control regulator $f(\xi)$ in (1.7) and (1.1) have the suboptimal three-positional structure (6.5) justified above. Then the following assertions hold:

(i) The controllability conditions (5.5) are necessary and sufficient for the existence of a feasible feedback control of type (6.5) to problem $(P)$ on any time interval $[0, T]$. More precisely, let $\gamma \beta-\eta>0$ and let $T \leq T_{0}$, where $T_{0}>0$ is the unique solution to the equation

$$
\beta\left(\gamma-\sum_{i=1}^{\infty} \frac{\mu_{i} \phi_{i}\left(x_{0}\right)}{\lambda_{i}} e^{-\lambda_{i} T_{0}}\right)=\eta .
$$

Then the trivial feedback control $f(\xi) \equiv 0$ on $\mathbb{R}$ (i.e., $v=0$ in (6.5), where $\sigma$ is thus irrelevant) is feasible by the state and control constraints in $(P)$ on the time interval $[0, T]$ and hence optimal to this problem.

(ii) Let further $T>T_{0}$ while the right-hand side inequality in (5.5) is fulfilled, let the recourse $v \in[0, \alpha]$ in (6.5) be computed by (5.7), and let

$$
\sigma(T):=\beta\left(\gamma-\sum_{i=1}^{\infty} \frac{\mu_{i} \phi_{i}\left(x_{0}\right)}{\lambda_{i}} e^{-\lambda_{i} \tau(T)}\right),
$$

where $\tau(T)$ is the unique solution to the transcendental equation (5.6). Then the feedback control (6.5) is feasible to $(P)$ on the time interval $[0, T]$ whenever $0<\sigma \leq \sigma(T)$ being in fact optimal to $(P)$ among regulators of this structure if $\sigma=\sigma(T)$.

(iii) The control recourse $v \in(0, \alpha]$ in (6.5) computed by formula (5.7) is necessary for stabilizing the observed trajectories $y\left(t, x_{0}\right)$ as $T \rightarrow \infty$ at one of the boundaries $\xi=\eta$ and $\xi=-\eta$ under the realization of the corresponding worst perturbations in the closed-loop control system (1.1), (1.7), and (6.5). Furthermore, we have $\sigma(T) \downarrow \bar{\sigma}$ as $T \rightarrow \infty$, where the number $\bar{\sigma} \geq 0$ is computed by

$$
\bar{\sigma}:=\beta\left(\gamma-\sum_{i=1}^{\infty} \frac{\mu_{i} \phi_{i}\left(x_{0}\right)}{\lambda_{i}}\left[\frac{\left(c+\lambda_{1}\right)(\gamma \beta-\eta)}{\beta(1+c \gamma)}\right]^{\frac{\lambda_{i}}{\lambda_{1}}}\right)
$$

and satisfies the following properties:

(a) $\bar{\sigma}=0$ if the extreme case (5.13) is realized in the controllability conditions (5.5). In this case the trivial feedback regulator $f(\xi) \equiv 0$ on $\mathbb{R}$ is feasible and thus optimal to $(P)$ on the infinite horizon $[0, \infty)$.

(b) $\bar{\sigma}>0$ if the strict inequality

$$
\gamma \beta-\eta<\frac{\beta(1+c \gamma)}{c+\lambda_{1}}
$$

holds in the controllability conditions (5.5). In this case the three-positional regulator (6.5) with the resource $v \in(0, \alpha]$ computed in $(5.7)$ is feasible to $(P)$ on $[0, \infty)$ whenever $0<\sigma \leq \bar{\sigma}$ being in fact optimal to $(P)$ on the infinite horizon $[0, \infty)$ when $\sigma=\bar{\sigma}$. 
Proof. A large part of the proof has been already given above and/or follows directly from the previous considerations. Indeed, the controllability conditions (5.5) ensure-by Theorem 5.1 for $(\widehat{P})$ and its symmetric counterparts for problem $(6.4)$ - the existence of feasible open-loop controls, which satisfy the control constraints (1.3) and keep the observed transients $y\left(t, x_{0}\right)$ within the state constraint region (1.5) under the realization of the worst system perturbations $w(t) \equiv \beta$ and $w(t) \equiv-\beta$ on $[0, T]$. Due to Theorem 3.1 on the monotonicity of transients with respect to controls (and thus with respect to switching times $\tau$ ) and also due to the time-monotonicity of $y(t)$ in (5.9), the feasible and optimal values of $\sigma$ asserted in the theorem directly relate, concerning the worst perturbations, to the corresponding values of $y(t)$ at $T_{0}$ and $\tau(T)$ determined in Theorem 5.1 and its proof. On the other hand, these values of $\sigma$ found for the case of the worst perturbations happen to be appropriate for any perturbations from the admissible area (1.4) due to the extremality of the worst perturbations by Theorem 3.2 and due to the monotonicity of transients with respect to perturbations by Theorem 3.1. In this way we arrive at all the conclusions of assertions (i) and (ii).

The conclusion on the control recourse $v$ in (iii) and also the statements in (a) are established in fact in the proof of Theorem 5.1. The value of $\bar{\sigma}$ in (6.8) corresponds to $\bar{\sigma}=y(\bar{\tau})$ with $y(t)$ from (5.9) and the asymptotically optimal switching time $\bar{\tau}$ computed by (5.8) due to the above arguments based on the monotonicity results of Theorem 3.1. The limiting conclusion $\sigma(T) \downarrow \bar{\sigma}$ as $T \rightarrow \infty$ can be checked directly, while all the statements in (b) follow from the above discussions due to the crucial transient monotonicity.

For further simplifications of the results obtained and also for the corresponding developments in the next Section 7, we impose the following assumption:

$$
\begin{aligned}
& \sum_{i=2}^{\infty} \frac{\mu_{i} \phi_{i}\left(x_{0}\right)}{\lambda_{i}} e^{-\lambda_{i} t}<0 \text { whenever } t=\frac{1}{\lambda_{1}} \ln \theta \text { with } \theta>1 \text {, i.e. } \\
& \sum_{i=2}^{\infty} \frac{\mu_{i} \phi_{i}\left(x_{0}\right)}{\lambda_{i}} \theta^{-\frac{\lambda_{i}}{\lambda_{1}}}<0 \text { whenever } \theta>1,
\end{aligned}
$$

which definitely holds for various standard parabolic equations in the presence of symmetry, e.g., for the multidimensional heat equation defined on rectangulars, balls, etc.; see, in particular, $[4,8,11]$ and the references therein.

Consider now the first term in the series (6.8), which is

$$
\bar{\sigma}_{1}:=\beta\left(\gamma-\frac{\mu_{1} \phi_{1}\left(x_{0}\right)\left(c+\lambda_{1}\right)(\gamma \beta-\eta)}{\lambda_{1} \beta(1+c \gamma)}\right)>0
$$

in the nontrivial case (6.9) of the controllability conditions (5.5). If in addition assumption (6.10) holds, then $\bar{\sigma}_{1}<\bar{\sigma}$, and hence the interval $\left[-\bar{\sigma}_{1}, \bar{\sigma}_{1}\right]$ lies entirely within the optimal dead region $(-\bar{\sigma}, \bar{\sigma})$ of the three-positional regulator $(6.5)$ by Theorem 6.1 (iii). Thus we arrive at the following consequence of the theorem.

Corollary 6.2 (first-order feasible approximation of optimal feedback control). In addition to the standing assumptions of Section 2, suppose that conditions (6.9) and (6.10) are satisfied. Then the three-positional feedback regulator $(6.5)$ with $v \in(0, \alpha]$ computed in (5.7) and with $\sigma=\bar{\sigma}_{1}$ computed in (6.11) is feasible to problem $(P)$ on the infinite horizon.

Proof. Follows from case (b) of Theorem 6.1(iii) due to $0<\bar{\sigma}_{1}<\bar{\sigma}$. 


\section{Robust Stability and Reliability of the Closed-Loop Con- strained Parabolic System}

The concluding section of the paper is devoted to the study of robust stability (or stability in the large) of the closed-loop control system

$$
\left\{\begin{array}{l}
\frac{\partial y}{\partial t}+A y=w(t), \quad x \in \Omega, \quad t \geq 0 \\
y(0, x)=0, \quad x \in \Omega, \\
y(t, x)=f\left(y\left(t, x_{0}\right)\right), \quad x \in \partial \Omega, \quad t \geq 0
\end{array}\right.
$$

where $f=f(\xi)$ is a three-positional feedback regulator with parameters $(v, \sigma)$ given in (6.5). Our goal is to derive efficient conditions ensuring the robust stability of system (7.1), (6.5) in the sense precisely defined below and then to combine these conditions with the relationships on $(v, \sigma)$ established in Section 6 from the viewpoint of controllability and minimax (sub)optimality in the feedback control problem $(P)$ for the parabolic system (7.1) subject to the control and state constraints. In this way we arrive at the reliable feedback control design ensuring the required suboptimal performance of the closed-loop control system in a stable regime acceptable for applications.

Note that the minimax design results of Section 6 establish relationships between parameters of the parabolic dynamics, feedback boundary controls, perturbations, and imposed constraints under which the closed-loop control system (7.1) allows us to keep all the transients at the point of observation within the prescribed state constraint region for any uncertain perturbations from the admissible area, with the optimal effect in the worst perturbation case. However, the above minimax control design does not address stability issues for the resulting closed-loop control system, which are of crucial importance for practical applications and are studied in detail in what follows.

We indicate the following two major sources that may cause possible instability of the closed-loop control system (7.1), (6.5):

(a) System (7.1) with $f(\xi)$ from (6.5) is highly nonlinear, despite the linearity of its parabolic dynamics. Of course, the source of nonlinearity is the discontinuous threepositional regulator (6.5) in the Dirichlet boundary conditions of (7.1).

(b) System (7.1) is of distributed parameters, which is the common name for control systems governed, in particular, by partial differential equations. In the framework of (7.1), the most significant manifestation of the distributed parameter nature is that the control acts in the boundary conditions while the feedback is formed by observing the current state position $\xi=y\left(t, x_{0}\right)$ at the intermediate point $x_{0} \in \Omega$ of the space domain. The latter generates the inertia/delay of the closed-loop control system and essentially affects stability.

One can easily see that if the current state position $\xi=y\left(t, x_{0}\right)$ lies inside the dead region $(-\sigma, \sigma)$ after terminating all the perturbations, then the closed-loop system (7.1) with the three-positional regulator (6.5) maintains the stationary equilibrium regime $y \equiv 0$ as $t \rightarrow \infty$. This signifies stability in the small of the initial equilibrium state $y \equiv 0$ in this system for any dead region $(-\sigma, \sigma)$ as $\sigma>0$. However, the latter property is not sufficient for the acceptable functioning of the nonlinear control system (7.1), (6.5) with distributed parameters. We need robust stability, or stability in the large, of the equilibrium state $y \equiv 0$ for the closed-loop system under consideration, which in our case means that $y\left(t, x_{0}\right) \rightarrow 0$ as $t \rightarrow \infty$ even if the current state $\xi$ of (7.1) is outside the dead region of (6.5) after terminating all the perturbations. The presence of perturbations $w(t)$ on some 
interval $[0, T]$ is clearly irrelevant to this stability issue, which is an internal property of the parabolic dynamics generated by the elliptic operator $A$ from (1.2) on the infinite horizon and the three-positional feedback regulator (6.5) in the Dirichlet boundary conditions of (7.1).

It has been well recognized in the literature that stability in the large (or robust stability) issues are among the most challenging in stability theory for nonlinear dynamics, even in the case of finite-dimensional control systems governed by ordinary differential equations. We are not familiar with any results in this direction for the parabolic systems studied in this paper. To derive efficient conditions for stability in the large of the equilibrium state $y \equiv 0$ in the closed-loop control system (7.1) with the three-positional feedback regulator (6.5), we develop a variational approach to such robust stability, which is largely based on the monotonicity properties of the parabolic dynamics and reduces the stability issue to solving an open-loop optimal control problem for the initial system (1.1) on the infinite horizon.

To proceed, observe from the structure of the closed-loop control system under consideration that the required robust stability of its stationary equilibrium state $y \equiv 0$ can be lost if the dead region in (6.5) is not sufficiently wide. Indeed, in such cases the transients $\xi=y\left(t, x_{0}\right)$ would move back and forth between the dead region boundaries under switching control positions in (6.5) with no external perturbations, just by inertia of the control system. This means that the closed-loop control system $(7.1),(6.5)$ may start functioning in a non-acceptable self-vibrating regime as $t \rightarrow \infty$ thus signifying instability in the large of the initial equilibrium stare $y=0$. We intend to find conditions that exclude such instability.

It follows from the above discussions that the unstable self-vibrating regime will not occur if the transient $y\left(t, x_{0}\right)$ starting at one boundary of the dead region does not reach the other boundary whenever $t>0$ under the control switching in (6.5) with no external perturbations. Moreover, the limiting stability resource of the system relates to the minimal width of the dead region ensuring the aforementioned property. This allows us to derive efficient stability conditions by solving an open-loop optimal control problem for (1.1) on the infinite horizon as is done in the proof of the next theorem.

Theorem 7.1 (robust stability of the closed-loop parabolic control system). Let (7.1) be a closed-loop parabolic system under the standing assumptions of Section 2, and let (6.5) be a three-positional feedback regulator in the boundary conditions of (7.1) with arbitrary parameters $v>0$ and $\sigma>0$. Then the control system (7.1), (6.5) exhibits robust stability in the above sense if its parameters satisfy the relationship

$$
\sigma \geq-\frac{v(1+c \gamma)}{2}+\frac{v+\sigma}{2} \sum_{i=1}^{\infty} \frac{\mu_{i} \phi_{i}\left(x_{0}\right)\left(c+\lambda_{i}\right)}{\lambda_{i}}\left(\frac{v}{v+\sigma}\right)^{\frac{\lambda_{i}}{\lambda_{1}}}
$$

where the right-hand side is always positive. If furthermore assumption (6.10) is fulfilled, then the stability condition can be simplified as

$$
\sigma \geq \frac{v}{2 \lambda_{1}}\left[\mu_{1} \phi_{1}\left(x_{0}\right)\left(c+\lambda_{1}\right)-\lambda_{1}(1+c \gamma)\right],
$$

where the right-hand side in (7.3) is always greater than the one in (7.2) whenever $v, \sigma>0$.

Proof. Developing a variational approach to robust stability, we consider the following open-loop control system on the infinite horizon:

$$
\left\{\begin{array}{l}
\frac{\partial y}{\partial t}+A y=0, \quad x \in \Omega, \quad t>0 \\
y(0, x)=0, \quad x \in \Omega, \\
y(t, x)=u(t), \quad x \in \partial \Omega, \quad t>0
\end{array}\right.
$$


with piecewise constant Dirichlet boundary controls given by

$$
u(t)= \begin{cases}h+\Delta h & \text { if } 0 \leq t \leq \tau \\ h & \text { if } t>\tau\end{cases}
$$

where $h$ and $\Delta h$ are some positive numbers (to be specified later) while $\tau$ is a control switching time to be determined. For formulating the other data (cost functional and state constraint) of the optimal control problem to study in what follows with the application to robust stability, we first employ Proposition 2.3 that gives the spectral representation of the transients $y(t, x)$ to $(7.4)$ generated by controls $(7.5)$. Since

$$
\sum_{i=1}^{\infty} \mu_{i} \phi_{i}(x)=1 \text { in } L^{2}(0, T) \text { and } \sum_{i=1}^{\infty} \frac{\mu_{i} \phi_{i}\left(x_{0}\right)}{\lambda_{i}}=\gamma
$$

we represent via (2.6) the corresponding solution to the boundary value problem in (7.4) and (7.5) at the point of observation $x=x_{0}$ as

$$
\begin{aligned}
y_{\tau}\left(t, x_{0}\right) & =\sum_{i=1}^{\infty} \mu_{i} \phi_{i}\left(x_{0}\right)\left(c+\lambda_{i}\right) e^{-\lambda_{i} t} \int_{0}^{t} u(\theta) e^{\lambda_{i} \theta} d \theta \\
& =\sum_{i=1}^{\infty} \mu_{i} \phi_{i}\left(x_{0}\right)\left(c+\lambda_{i}\right) e^{-\lambda_{i} t}\left(\int_{0}^{\tau}(h+\Delta h) e^{\lambda_{i} \theta} d \theta+\int_{\tau}^{t} h e^{\lambda_{i} \theta} d \theta\right) \\
& =(1+\gamma c) h+\sum_{i=1}^{\infty} \frac{\mu_{i} \phi_{i}\left(x_{0}\right)\left(c+\lambda_{i}\right)}{\lambda_{i}}\left[\Delta h e^{\lambda_{i} \tau}-(h+\Delta h)\right] e^{-\lambda_{i} t}
\end{aligned}
$$

It is easy to see from (7.6) that

$$
y_{\tau}\left(t, x_{0}\right) \rightarrow(1+c \gamma) h \text { as } t \rightarrow \infty \text { whenever } \tau>0
$$

However, the transient $y\left(t, x_{0}\right)$ may intersect the stabilization level $(7.7)$ if the switching time $\tau$ is not properly chosen. We intend to find efficient conditions under which the latter situation does not occur. These conditions, being of certain interest for their own sake, ensure the required robust stability of the closed-loop system (7.1), (6.5) when the control levels $h$ and $\Delta h$ in (7.5) are specified appropriately.

To proceed, consider the following auxiliary dynamic optimization problem for the parabolic system (7.4) on the infinite horizon:

$$
\left\{\begin{array}{l}
\text { minimize } J(\tau):=(1+c \gamma) h-y_{\tau}\left(\tau, x_{0}\right) \\
\text { subject to }(7.4),(7.5), \text { and the state constraint } \\
y_{\tau}\left(t, x_{0}\right)<(1+c \gamma) h \text { for all } t>0
\end{array}\right.
$$

The meaning of this problem is to find an optimal switching time $\tau=\tau>0$ in (7.5) such that the corresponding trajectory $y_{\underline{\tau}}\left(t, x_{0}\right)$ to $(7.4)$ lies strictly below the stabilization level (7.7) for all $t>0$ (i.e., does not reach this level whenever $t>0$ ) and that the distance between the stabilization level (7.7) and the switching level

$$
y\left(\underline{\tau}, x_{0}\right):=y_{\underline{\underline{\tau}}}\left(\underline{\tau}, x_{0}\right)
$$

is minimal in comparison with any other switching time $\tau$ satisfying all the constraints in (7.8). According to the discussions on robust stability presented right before the formulation 
of Theorem 7.1, solving this optimal control problem directly leads us to required stability conditions; see below for more details.

It follows from the monotonicity property of Theorem 3.1 with respect to controls that

$$
y_{\tau_{1}}\left(t, x_{0}\right) \leq y_{\tau_{2}}\left(t, x_{0}\right) \text { whenever } t>0 \text { and } \tau_{1} \leq \tau_{2}
$$

for the transients $y_{\tau}\left(t, x_{0}\right)$ generated in (7.6) by the switching controls (7.5). Thus the optimal switching time $I$ to (7.8) is the largest one under which the corresponding transient $y_{\tau}\left(t, x_{0}\right)$ does not intersect the stabilization level $(1+c \gamma) h$ for all $t>0$.

The exact solution to the open-loop control problem (7.8) on the infinite horizon is given in Theorem 5.1(ii)-more precisely, in its proof. It is provided by the first term rule, i.e., by vanishing the first term in the last series of (7.6). By this result we have the simple (while rigorously justified) formula for the optimal switching time to (7.8):

$$
\tau=\frac{1}{\lambda_{1}} \ln \left(\frac{h+\Delta h}{\Delta h}\right)>0 \text { whenever } v, \sigma>0,
$$

and hence the exact optimal value of the cost functional in this problem is computed by:

$$
\begin{aligned}
\underline{\vartheta}: & =J(\underline{\tau})=(1+c \gamma) h-y\left(\underline{\tau}, x_{0}\right) \\
& =-\Delta h(1+c \gamma)+(h+\Delta h) \sum_{i=1}^{\infty} \frac{\mu_{i} \phi_{i}\left(x_{0}\right)\left(c+\lambda_{i}\right)}{\lambda_{i}}\left(\frac{\Delta h}{h+\Delta h}\right)^{\frac{\lambda_{i}}{\lambda_{1}}}>0 .
\end{aligned}
$$

Imposing assumption (6.10), in addition to the standing hypotheses of Section 2, we get the feasible first-order approximation

$$
\underline{\vartheta}_{1}:=\Delta h\left[\frac{\mu_{1} \phi_{1}\left(x_{0}\right)\left(c+\lambda_{1}\right)}{\lambda_{1}}-(1+c \gamma)\right]>\underline{\vartheta}>0
$$

to (7.9), which happens to be independent of the control level $h$ in (7.5).

According to the description of the instability (in the large) phenomenon given before the formulation of Theorem 7.1, robust stability of the closed-loop control system (7.1), (6.5) is ensured if the width of the dead region $2 \sigma$ is not smaller than the value $\underline{\vartheta}$ in (7.9) with $h=\sigma$ and $\Delta h=v$. Substituting these data into (7.9), we arrive at the stability condition (7.2) of the theorem. The explicit first-order approximation condition (7.3) corresponds to substituting the above values of $h$ and $\Delta h$ into formula (7.10) for $\underline{\vartheta}_{1}$ via the sufficient stability requirement $2 \sigma \geq \underline{\vartheta}_{1}$. This completes the proof of the theorem.

Finally, we combine the feedback control results derived in Section 6 from the viewpoint of controllability and minimax optimality with the robust stability conditions obtained in this section; thus we establish reliability relationships between all the parameters of the feedback control, parabolic dynamics, imposed constraints, and perturbations that ensure feasible and then (sub)optimal behavior of the closed-loop control system under consideration in a stable regime. Since the control resource $v$ in (6.5) is uniquely determined by (5.7), the remaining issue is to justify the existence of the reliable dead region $(-\sigma, \sigma)$ in (6.5) and to describe further the reliability range of the acceptable variety for the characteristic parameter $\sigma$ of the feedback regulator.

Theorem 7.2 (reliability of the feedback control design). Consider the closed-loop control parabolic system (7.1) with uncertain perturbations $w \in W$ from (1.4) and with the there-positional feedback regulator (6.5) in the Dirichlet boundary conditions. Assume that 
the standing hypotheses of Section 2 and the controllability conditions (5.5) are satisfied. The following assertions hold:

(i) Let the extreme case (5.13) be realized in the controllability conditions (5.5). Then the trivial feedback regulator $f(\xi) \equiv 0$ in (6.5) ensures both robust stability and optimality to the minimax problem $(P)$.

(ii) Let the controllability conditions (5.5) hold excluding the extreme case (5.13), and let the control resource $v \in(0, \alpha]$ in (6.5) be computed by (5.7). Denote

$$
\begin{aligned}
\underline{\sigma}: & =\frac{\gamma \beta-\eta+\eta(1+c \gamma)}{2(1+c \gamma)} \sum_{i=1}^{\infty} \frac{\mu_{i} \phi_{i}\left(x_{0}\right)\left(c+\lambda_{i}\right)}{\lambda_{i}}\left(\frac{\gamma \beta-\eta}{\gamma \beta-\eta+\eta(1+c \gamma)}\right)^{\frac{\lambda_{i}}{\lambda_{1}}} \\
& +\frac{\eta-\gamma \beta}{2}>0
\end{aligned}
$$

and suppose that $\underline{\sigma} \leq \bar{\sigma}$, where $\bar{\sigma}>0$ is computed by (6.8). Then the feedback control system (7.1), (6.5) with the dead region parameter $\sigma>0$ belonging to the nonempty interval

$$
\underline{\sigma} \leq \sigma \leq \bar{\sigma}
$$

is reliable on the infinite horizon in the sense that it is feasible by all the constraints in $(P)$ on $[0, \infty)$ for any perturbations $w \in W$ enjoying simultaneously robust stability. Furthermore, the upper bound $\sigma=\bar{\sigma}$ of the reliable range (7.12) optimizes the suboptimal feedback structure $(6.5)$ to the minimax problem $(P)$ on $[0, \infty)$ under the worst perturbations.

(iii) Suppose in addition to the hypotheses in (ii) that the first-order approximation assumption (6.10) is fulfilled. Denote

$$
\underline{\sigma}_{1}:=\frac{\gamma \beta-\eta}{2 \lambda_{1}(1+c \gamma)}\left[\mu_{1} \phi_{1}\left(x_{0}\right)\left(c+\lambda_{1}\right)-\lambda_{1}(1+c \gamma)\right]>\underline{\sigma}
$$

and impose further the first-order reliability condition

$$
3 \mu_{1} \phi_{1}\left(x_{0}\right)\left(c+\lambda_{1}\right)(\gamma \beta-\eta) \leq \lambda_{1}(3 \gamma \beta-\eta)(1+c \gamma) .
$$

Then $\underline{\sigma}_{1} \leq \bar{\sigma}_{1}$, where $\bar{\sigma}_{1} \in(0, \bar{\sigma})$ is given in $(6.11)$, and the feedback control system (7.1), (6.5) is reliable on the infinite horizon in the sense described in (ii) with the contral resource $v \in(0, \alpha]$ from (5.7) and the dead region parameter $\sigma>0$ satisfying

$$
\underline{\sigma}_{1} \leq \sigma \leq \bar{\sigma}_{1} \text {. }
$$

Furthermore, the reliability condition (7.14) can be equivalently described directly via the suboptimal first-order value $\bar{\sigma}_{1}$ from (6.11) as $\bar{\sigma}_{1} \geq \eta / 3$.

Proof. This theorem unifies and summarizes, to a large extent, the feedback control design results derived above. To begin with, observe that assertion (i) follows directly from case (a) of Theorem 6.1(iii), since the closed-loop system (7.1) with the trivial regulator $f(\xi) \equiv 0$ in (6.5) obviously exhibits robust stability (no control switching).

To justify assertion (ii), we apply Theorem 7.1 in order to check the robust stability of system (7.1) with the three-positional regulator (6.5), where $v$ is computed by (5.7)-due to case (b) of Theorem 6.1(iii) - and where $\sigma \geq \underline{\sigma}$ with $\underline{\sigma}$ computed by (7.11). This follows from the observation that the value of $\underline{\sigma}$ in (7.11) is in fact obtained by substituting $v$ from (5.7) into the right-hand side of (7.2) and by replacing $\sigma$ with $\eta$ therein. We easily conclude 
that $\underline{\sigma}$ satisfies inequality (7.2) whenever $0<\sigma \leq \eta$ in the right-hand side of it, which is the case under consideration. The other statements in (ii) are proved in Theorem 6.1(iii).

To justify assertion (iii), observe first that the value of $\underline{\sigma}_{1}$ in (7.13) is obtained by substituting $v$ from (5.7) into the right-hand of (7.3). Furthermore, the first-order reliability condition (7.14) is directly derived from the condition $\underline{\sigma}_{1} \leq \bar{\sigma}_{1}$ by substituting there $\bar{\sigma}_{1}$ from (6.11) and $\underline{\sigma}_{1}$ from (7.13). Thus the feasibility of the three-positional regulator (6.5) with $v$ from (5.7) and $\sigma$ from (7.15) follows from Corollary 6.2, while the corresponding robust stability of system (7.1), (6.5) with $\sigma$ in (7.15) follows from the last part of Theorem 7.1. Finally, we can directly check that the reliability condition (7.14)-ensuring robust stability - can be surprisingly rewritten in the very simple form $\bar{\sigma}_{1} \geq \eta / 3$ via just the first-order suboptimality value $\bar{\sigma}_{1}$ computed by (6.11). Note that the equality therein can be used as an additional equation for shape optimization to determine, e.g., the best parameters of the domain $\Omega$ ensuring a reliable feedback control design under the other given data of the minimax problem $(P)$ and the feedback regulator (6.5).

Acknowledgment. The author is grateful to Jean-Pierre Raymond and Tom Seidman for helpful discussions and remarks on the material of this paper.

\section{References}

[1] N. ARADa AND J.-P. RAYMOND, Dirichlet boundary control of semilinear parabolic equations, Part 1: problems with no state constraints, Appl. Math. Optim., 45 (2002), pp. $125-143$.

[2] T. Başar And P. Bernhard, $H_{\infty}$-Optimal Control and Related Minimax Design Problems, Birkhäuser, Boston, MA, 1995.

[3] H. O. FATTORINI AND T. MURPHY, Optimal problems for nonlinear parabolic boundary control systems, SIAM J. Control Optim., 32 (1994), 1577-1596.

[4] D. Gilbarg And N. S. Trudinger, Elliptic Partial Differential Equations of Second Order, Springer, Berlin, 1983.

[5] A. D. Ioffe and V. M. Tikhomirov, Theory of Extremal Problems, North-Holland, Amsterdam, 1979.

[6] B. van Keulen, $H_{\infty}$-Control for Distributed Parameter Systems: A State-Space Approach, Birkhäuser, Boston, MA, 1993.

[7] N. N. Krasovskin and A. I. Subbotin, Game-Theoretical Control Problems, Springer, New York, 1988.

[8] O. A. Ladyzhenskaya, A. I. Solonnikov and N. N. URaltseva, Linear and Quasilinear Equations of Parabolic Type, American Mathematical Society, Providence, RI, 1968.

[9] I. Lasiecka and R. Triggiani, Control Theory for Partial Differential Equations: Continuous and Approximation Theory, published in two volumes, Cambridge University Press, Cambridge, UK, 2000. 
[10] J.-L. LIons, Optimal Control of Systems Governed by Partial Differential Equations, Springer, Berlin, 1971.

[11] S. Mizohata, The Theory of Partial Differential Equations, Cambridge University Press, Cambridhe, UK, 1973.

[12] B. S. MoRdukHovich, Optimal control of the groundwater regime on engineering reclamation systems, Water Resources, 12 (1986), pp. 244-253.

[13] B. S. MordukHovich, Minimax design for a class of distributed parameter systems, Autom. Remote Control, 50 (1990), pp. 262-283.

[14] B. S. MoRdukHovich, Variational Analysis and Generalized Differentiation, II: Applications, Grundlehren Series (Fundamental Principles of Mathematical Sciences), Vol. 331, Springer, Berlin, 2006.

[15] B. S. MoRDUKHovich, Suboptimal minimax design of constrained parabolic systems with mixed boundary controls, Appl. Math. Comput., 204 (2008), pp. 580-588.

[16] B. S. MoRduKHovich, Optimization and feedback design of state-constrained parabolic systems, Pacif. J. Optim., 4 (2008), 549-570.

[17] B. S. Mordukhovich and T. I. SeIDMan, Asymmetric games for convolution systems with applications to feedback control of constrained parabolic equations, J. Math. Anal. Appl., 333 (2007), pp. 401-415.

[18] B. S. Mordukhovich AND I. ShVARTSMan, Optimization and feedback control of constrained parabolic systems under uncertain perturbations, in Optimal Control, Stabilization and Nonsmooth Analysis (M. de Queiroz et al., eds.), Lecture Notes Cont. Inf. Sci., vol. 301, pp. 121-132, Springer, New York, 2004.

[19] B. S. MoRdukHovich AND K. ZHANG, Minimax control of parabolic equations with Dirichlet boundary conditions and state constraints, Appl. Math. Optim., 36 (1997), pp. $323-360$.

[20] L. S. Pontryagin, V. G. Boltyanskil, R. V. Gamkrelidze and E. F. Mishchenko, The Mathematical Theory of Optimal Processes, Wiley, New York, 1962.

[21] J.-P. RAYMOND AND H. ZIDANI, Pontryagin's principle for state-constrained control problems governed by parabolic equations with unbounded controls, SIAM J. Control Optim., 38 (1998), 1853-1879. 